Nouvelles perspectives en sciences sociales

Revue internationale de systémique complexe et d'études relationnelles

\title{
Une mesure unifiée de la complexité linguistique : l'analyse matricielle définitoire
}

\section{Amr Helmy Ibrahim}

Volume 9, numéro 1, novembre 2013

Sur le thème : linguistique et complexité

URI : https://id.erudit.org/iderudit/1024038ar

DOI : https://doi.org/10.7202/1024038ar

Aller au sommaire du numéro

Éditeur(s)

Prise de parole

ISSN

1712-8307 (imprimé)

1918-7475 (numérique)

Découvrir la revue

Citer cet article

Ibrahim, A. H. (2013). Une mesure unifiée de la complexité linguistique :

l'analyse matricielle définitoire. Nouvelles perspectives en sciences sociales,

9(1), 17-80. https://doi.org/10.7202/1024038ar
Résumé de l'article

Cet article essaie de démontrer qu'il est possible d'avoir une mesure unifiée et standardisée de la plupart des types de complexité linguistique quelle que soit la langue à condition de recourir à un système de description et d'interprétation du fonctionnement des langues - en l'occurrence l'analyse matricielle définitoire (AMD) - qui s'appuie sur cette propriété remarquable des langues naturelles de pouvoir se décrire avec leurs termes les plus courants à travers la construction de classes d'équivalence au sein desquelles les faits grammaticaux - principale source de complexité - sont " décomplexifiés » et mesurés en termes d'opérations régulières de réduction. 


\title{
Une mesure unifiée de la complexité linguistique : l'analyse matricielle définitoire
}

\author{
Amr Helmy Ibrahim \\ Université de Franche-Comté (Besançon) \\ et Université de Paris-Sorbonne
}

\section{Péréquation des complexités d'une langue à l'autre}

$\mathbf{U}$

ne langue que nous ne connaissons pas ou que nous mầtrisons mal nous paraîtra toujours plus complexe qu'une langue que nous connaissons ou avec laquelle nous sommes familiers. La pudeur académique rebaptisera cette complexité-là "difficulté », mais cela ne change rien à l'affaire. De fait, et sans sous-estimer l'importance des jugements subjectifs des locuteurs dans leur comportement linguistique, il existe des mesures plus ou moins objectives de la complexité d'un objet qui ne doivent rien au degré de connaissance ou d'ignorance qu'on en a.

Les descriptions montrent des différences de complexité entre les langues, non pas au sens où une langue, prise dans sa totalité, serait plus ou moins complexe qu'une autre, mais au sens où le parcours d'une langue pour rendre compte d'une représentation particulière est plus complexe que le parcours d'une autre pour rendre compte de la même représentation. Si les langues, prises une à une et dans la totalité de leurs possibilités expressives, sont également complexes du fait d'une sorte de péréquation ou de 
compensation entre leurs parcours plus complexes et leurs parcours moins complexes, elles peuvent présenter sur l'expression d'un point particulier, envisagé à partir d'un point de vue particulier, des degrés différents de complexité.

$\mathrm{Si}$, à titre d'exemple, on compare les outils d'ancrage temporel du point de vue de l'ordre de la succession des moments d'un processus, dans une langue comme le français, aux outils qui visent à atteindre les mêmes objectifs dans une langue comme l'arabe ou le polonais, on constatera la disponibilité en français d'une profusion de formes susceptibles de s'opposer et de se combiner sur des axes qui privilégient, pour exprimer toutes sortes de valeurs temporelles mais aussi aspectuelles, énonciatives ou modales, la représentation d'un ancrage dans des moments et dans la succession de ces moments. Parallèlement, on constatera, à première vue, une pauvreté, relative en polonais et flagrante en arabe, de ces mêmes outils, c'est-à-dire des formes dédiées à signaler comment des moments donnés s'ancrent et se succèdent dans le temps, même si un examen plus approfondi du système aspectuel de ces deux langues et de la distribution de leurs morphèmes préverbaux montrera qu'elles ont recours à d'autres outils pour parvenir au même résultat. On a alors non seulement affaire à une différence de complexité, mais aussi de type de complexité.

L'usage du français exigera de choisir le bon outil - une forme morphophonémique formée en priorité pour rendre compte de l'ancrage temporel mais qui, en fait, réunit quatre variables paradigmatiques - le temps, le mode, la voix et l'aspect - parmi de nombreux candidats possibles : une trentaine de formes pour chaque personne. La difficulté dans cette langue sera de savoir faire le tri entre toutes les formes susceptibles d'exprimer ce que l'on veut dire puis, face à une combinatoire a priori ouverte et presque infinie, de trouver la combinaison pertinente pour la cohérence du discours que l'on tient et la perspective dans laquelle on le tient.

En polonais, la difficulté sera d'un tout autre type. Les outils dédiés spécifiquement à l'expression de l'ancrage temporel et à la 
succession des moments dans le temps sont moins nombreux et moins performants, mais leur action peut être complétée avec les nombreux outils dédiés à l'expression de l'aspect et du mode d'action (Aktionsart). Il faut donc savoir faire le tri entre un grand nombre d'outils à finalité hétérogène et dont les règles de combinaison sont, elles aussi, hétérogènes.

En arabe il n'existe tout simplement pas d'outils dédiés spécifiquement à l'expression de l'ancrage temporel et à la succession des moments dans le temps en dehors des morphèmes préverbaux sa et sawfa dédiés à l'expression du futur. Il faudra donc, par une procédure qui n'est pas sans rappeler l'exaptation ${ }^{1}$ postulée par Stephen Jay Gould et Elizabeth S. Vrba ${ }^{2}$ pour expliquer l'évolution des espèces, détourner les outils qui sont dédiés en arabe à l'expression de l'aspect et du mode d'action pour exprimer l'ancrage temporel et la succession des moments dans le temps. Ces outils étant peu nombreux, la procédure sera à la fois adaptative et combinatoire ${ }^{3}$.

$\mathrm{Si}$ ces constatations sont correctes, il y a, selon les secteurs grammaticalisés d'une langue - expression du temps, de l'enchaînement, de la quantité, de la causalité, du mode d'action, etc. -, différents types de complexité, et il y a aussi, dans chaque langue, différentes manières de compenser les disparités entre le degré de complexité de chaque secteur de la grammaire. Il convient donc d'avoir un étalonnage des paramètres de la complexité qui donne, pour chaque secteur grammatical, une mesure transposable aux autres secteurs de la même langue, mais aussi des autres langues, puisque la péréquation entre les

1 «Le fait qu'une propriété d'un organisme soit cooptée pour un usage pour lequel elle n'a pas été modifiée au cours du processus évolutif d'adaptation " (Traduction française adaptée d'un passage de l'Encyclopédie en ligne de l'Université de Stanford http://plato.stanford.edu/entries/teleology-biology/ à l'entrée Adaptation, Exaptation and Co-opted Use ).

2 Stephen Jay Gould et Elizabeth S. Vrba, "Exaptation - A missing term in the science of form ", Paleobiology, vol. 8, n 1, 1982, p. 4-15.

3 Voir Amr Helmy Ibrahim, Étude comparée des systèmes verbaux de l'arabe égyptien, de l'arabe moderne et du français, Thèse de doctorat d'État, Université de Paris, 1979 et "Le futur : une notion temporelle que les langues construisent avec de l'aspect ", à paraître. 
complexités des différents secteurs d'une même langue n'est appréciable qu'en comparaison avec les mesures obtenues dans une autre langue.

\section{Première étape de la mesure : l'étalonnage de la simplicité}

Une mesure, même relative, de la complexité doit partir d'un étalonnage de la simplicité. Mais la simplicité en langue n'est pas la même selon qu'il s'agit d'une simplicité d'usage qui a recours à une forme préconstruite, qui peut être le résultat d'une formation complexe, ou d'une simplicité intrinsèque, une forme à la fois indécomposable et facile d'accès. Si l'on ne se réfère qu'à la simplicité d'accès à la forme dans l'usage, c'est une forme qui constitue le plus grand dénominateur commun à l'éventail le plus large de formes complexes courantes. Ce dénominateur commun peut être strictement statistique - le plus grand dénominateur commun au plus grand nombre de formes - comme il peut être la limite inférieure d'une décomposition ou être un mixte des deux. C'est la raison pour laquelle il n'y a probablement pas de sens à parler, dans l'usage, de simplicité formelle si l'on ne connaît pas les modalités d'accès aux usages hérités. L'usage peut rendre une forme intrinsèquement complexe plus directement accessible qu'une forme intrinsèquement simple.

De plus, la simplicité n'est pas nécessairement envisagée de la même manière quand on se situe dans l'économie du système grammatical. Dans ce cas, la forme la plus simple est, indépendamment de l'accessibilité de son usage ou de sa fréquence, la forme la moins contrainte grammaticalement et comme les contraintes grammaticales sont réputées cristalliser toutes les formes de complexité, la mesure de la levée ordonnée des contraintes grammaticales est aussi une mesure de la complexité ou, si l'on préfère, du nombre et de la nature des opérations nécessaires pour passer d'une forme complexe à une forme simple, c'est-à-dire dégrammaticalisée.

Mais cette mesure ne rend pas compte à elle seule de toutes les relations grammaticales du fait que certaines relations au sein de la grammaire ne sont pas purement combinatoires et ne 
peuvent pas de ce fait être généralisées mécaniquement. C'est le cas notamment des relations d'appropriation. Ainsi certaines associations ou cooccurrences de mots ne sont pas commandées uniquement par la catégorie à laquelle elles appartiennent, mais par une forme de compatibilité à mi chemin de la contrainte syntaxique et de la contrainte sémantique. Ainsi on n'a pas en français *attraper la fuite mais prendre la fuite, on n'a pas * serrer une relation, mais nouer une relation, on n'a pas *posséder un sentiment, mais avoir ou éprouver un sentiment, on n'a pas *filer un risque mais courir un risque. Parallèlement, le français dira on est resté trois heures et non ${ }^{*}$ on a resté trois heures, mais utilisera indifféremment les deux auxiliaires pour Il a disparu ou Il est disparu alors qu'il dira Il a disparu sans crier gare mais jamais *Il est disparu sans crier gare.

Les relations d'appropriation ont ceci de particulier qu'elles relient des constituants indécomposables, donc simples, mais que les règles qui relient ces constituants au sein du groupe ainsi constitué ne sont pas celles auxquelles seraient soumis les mêmes constituants s'ils étaient reliés non par une relation d'appropriation mais par une relation ouverte d'opérateur à opérande : dans donner une gifle dont le sens est absolument identique à gifler le verbe donner et le nom gifle sont dans une relation réciproque d'appropriation qui construit un paradigme relativement homogène sémantiquement; on trouve donner dans une relation comparable avec coup dans, par exemple : donner (un coup de pied + un coup de poing + une raclée + une fessée + une claque...). Cette relation est très différente de celle qui associe donner à livre, gâteau ou voiture, qui appartiennent, eux, à un paradigme sémantiquement ouvert. Cette différence de nature dans la construction de la relation entre le verbe donner et un nom selon qu'ils sont dans une relation d'appropriation ou de combinatoire ouverte est ${ }^{4}$ une source importante de complexité bien qu'elle touche des constituants terminaux simples, c'est-à-dire indécomposables. Mais l'analyse de la généalogie des formes n'est pas la même dans les deux cas. On ne dégrammaticalise pas les énoncés

$\overline{4 \quad \text { Voir la section }}$ 5.3.2. 
de la même manière selon que l'on a affaire à une construction à verbe distributionnel (donner un livre) ou à verbe support (donner une gifle). L'analyse de la complexité qui comporte une relation d'appropriation comme dans donner une gifle exige un passage par la grammaire du prédicat nominal gifle donc par la relation gifle>gifler, par le paradigme complet des verbes supports actualisateurs de ce prédicat : donner, asséner, flanquer, ficher, foutre... ainsi que par celui des verbes impliqués dans la relation converse : écoper de, recevoir, par le type de détermination induit par la relation d'appropriation: Quand il lui a donné (le +un) diamant, elle a pleuré mais Quand il lui a donné (*la + une) gifle, elle a pleuré où l'on voit que la relation d'appropriation bloque le renvoi anaphorique, enfin par la participation des constructions figées ou semi-figées impliquant gifle dans la structuration du champ lexical du prédicat : des expressions comme tête à claques / tête à gifles ${ }^{5}$ dont la vitalité et l'actualité découle directement d'une matrice du genre : c'est le type de personne qui vous donne envie quand vous la voyez de lui ficher (une paire de gifles + une paire de claques). Cette forme de complexité est sans objet dans le cas d'une relation distributionnelle du type de celle que l'on a dans Il lui a donné un diamant.

Étant donné ces différentes formes de complexité, on peut poser que l'étalon de la simplicité se confond avec les produits terminaux indécomposables de l'analyse, à savoir les constituants que l'on retrouve dans une matrice analytique définitoire complète $^{6}, c^{\prime}$ est-à-dire une matrice dont aucun constituant n'est plus décomposable en constituants plus élémentaires. La mesure de la complexité devient alors la mesure du nombre et de la nature des opérations et des étapes qui mènent de la matrice complète à l'énoncé observé.

\footnotetext{
5 "Elle avait une tête à gifles. - Vous venez? lui dis-je en descendant de voiture (...) Dépêchez-vous, je suis pressé. " (Cendrars, L’homme foudroyé [Cité par le TLF])

6 Voir la section 4.
} 


\section{Complexité préconstruite et complexité à construire}

Les opérations qui aboutissent à la complexification des constituants terminaux simples peuvent suivre deux parcours distincts.

(1) Un parcours dont on est conscient et qui vient d'une succession de choix parmi un éventail de combinaisons possibles. Cette première forme de complexité exige, d'une part, de choisir des combinaisons qui mènent à un résultat et, d'autre part, de disposer d'un certain nombre de critères de référence pour effectuer ces choix: Je pourrais l'appeler tout de suite mais je ne suis pas sûr qu'il puisse nous donner une réponse qui vous satisfasse / Je peux l'appeler tout de suite mais je ne suis pas convaincu qu'il répondra d'une manière que vous jugerez satisfaisante / On peut tout à fait l'appeler maintenant sauf que je ne peux pas vous garantir une réponse satisfaisante / Je l'appelle immédiatement. Mais ça ne veut pas dire que vous aurez la réponse que vous attendez / etc. Ces énoncés, et on est loin d'avoir fait le tour de toutes les variations possibles à l'intérieur d'une fourchette très étroite de significations possibles dans le cadre d'un échange dont on peut facilement reconstituer les conditions pragmatiques, impliquent de nombreux choix à l'intérieur d'une gamme récurrente de possibilités. Ces choix génèrent une complexité qui fait penser à celle à laquelle est confronté le joueur d'échecs sommé de suivre une constante tout en s'adaptant aux mouvements de l'autre.

(2) En plus de cette complexité explicite faite de choix, il y a dans la langue une deuxième forme de complexité dont le locuteur n'est le plus souvent pas conscient. Il s'agit d'une complexité pour ainsi dire préconstruite, sous-jacente aux formes plus ou moins fortement grammaticalisées de la langue et qui a généralement tous les dehors de la simplicité. Du fait de la fréquence de son usage ou du fait que cet usage n'implique pas de choisir entre plusieurs formes possibles dans le même contexte, elle est perçue comme simple. Sa complexité réside dans le parcours qui l'a rendue disponible, dans sa généalogie. La description de la langue montre que l'accès à cette complexité sous-jacente, même quand il est dans les faits très rapide, reste opaque. Les formes qui relèvent de cette complexité ont une apparence 
simple, élémentaire voire première et indécomposable. Elles sont en fait décomposables7. Ainsi par exemple l'expression: Assis! a-t-elle une généalogie fort complexe de type : Je (te + vous) (demande + ordonne) de (t'asseoir + vous asseoir + rester assis)!

\section{Comment procède l'analyse matricielle définitoire?}

L'analyse matricielle définitoire ${ }^{8}$, avec laquelle nous proposons d'étalonner les différents niveaux de complexité, consiste à analyser, sous la forme d'une paraphrase définitoire et sans recours à un quelconque métalangage, toutes les relations linguistiques entre un énoncé observé qu’on peut qualifier de synthétique et sa matrice, c'est-à-dire son équivalent analytique maximal. Ainsi Mange! aura-t-il pour matrice maximale : Une personne qui ne se désigne pas dans son énoncé dit en adoptant une intonation d'ordre à une personne non désignée dans l'énoncé de procéder à un moment immédiatement après le moment de son énonciation à l'ingestion de quelque chose de mangeable.

Cette matrice est le produit d'une décomposition en plusieurs étapes de Mange! :

(1) Étiquetage du premier actant ou premier argument : une personne;

(2) Détermination du premier actant ou premier argument : qui ne se désigne pas dans son énoncé;

(3) Application de l'opérateur métaphrastique : dire;

(4) Application de l'opérateur prosodique : intonation d'ordre;

(5) Introduction du repère temporel de l'énonciation : le moment de son énonciation;

$\overline{7}$ Cette forme de complexité a beaucoup de points communs avec la simplexité définie par Alain Berthoz, La simplexité, Paris, Odile Jacob.

8 Pour un exposé plus détaillé du cadre théorique et méthodologique de l'AMD (Analyse matricielle définitoire) voir Amr Helmy Ibrahim, "Prolégomènes à une typologie de l'actualisation des noms ", dans Jacques François et Imtraud Behr (dir.), Les constituants prédicatifs et la diversité des langues, Mémoires de la Société de Linguistique de Paris, Tome XIV, 2004, p. 29-76; "Les conditions de la prédication dans les langues ", Prédicats, prédication et structures prédicatives, Paris, CRL, 2009, p. 12-49; "Supports d'actualisation et dualité constitutive de la prédication ", Supports et prédicats non verbaux dans les langues du monde, Paris, CRL, 2010, p. 36-73; L'analyse matricielle définitoire : un modèle pour la description et la comparaison des langues, à paraître. 
(6) Introduction du repère temporel du foyer prédicatif : un moment;

(7) Application de l'opérateur de succession temporelle : après; (8) Application de l'opérateur de perfectivation : immédiatement;

(9) Étiquetage du $2^{\mathrm{e}}$ actant ou $2^{\mathrm{e}}$ argument : une personne; (10) Détermination du $2^{\mathrm{e}}$ actant ou $2^{\mathrm{e}}$ argument : non désignée dans l'énoncé;

(11) Décomposition du prédicat en : procéder à l'ingestion de quelque chose de mangeable.

4.1. Analyser des changements dans le cadre d'une équivalence

L'analyse matricielle définitoire est fondamentalement une analyse des relations d'équivalence et des modifications que subissent les constituants des énoncés impliqués dans ces relations d'équivalence qui sont aussi des formes de redondances. Cet impératif d'équivalence joue un double rôle. D'une part, il sélectionne les modifications et les relations que l'on est en droit d'accepter ou de refuser; d'autre part, il constitue un repère absolu par rapport auquel il sera possible de mesurer toute sorte de variation qu'il s'agisse de la modification d'un constituant, de son insertion ou de son effacement - sa réduction?

$9 \quad$ Nous utilisons à ce stade la notion d'équivalence dans le sens précis qu'Antoine Laurent de Lavoisier a donné en 1789 à l'opération « une des plus frappantes et des plus extraordinaires de toutes celles que la chimie nous présente ", " de la décomposition des oxydes végétaux par la fermentation vineuse " lorsqu’il observe " comment un corps doux, un oxyde végétal, peut se transformer [...] en deux substances si différentes - le gaz acide carbonique et la liqueur vineuse - dont l'une est combustible, l'autre éminemment incombustible ». Opération dont il a tiré la célèbre conclusion, fondement indiscutable de bon nombre de pratiques scientifiques qu'il s'agisse de la méthodologie des expériences ou de la conceptualisation de leurs résultats : "rien ne se crée dans les opérations de la nature, et l'on peut poser en principe que dans toute opération, il y a une égale quantité de matière avant et après l'opération; que la qualité et la quantité des principes est la même, et qu'il n'y a que des changements, des modifications ». Et il ajoute : "C'est sur ce principe qu'est fondé tout l'art de faire des expériences en chimie : on est obligé de supposer dans toutes une véritable égalité ou équation entre les principes d'un corps qu'on examine et ceux qu'on en retire par l'analyse ". 
De fait, les relations d'équivalence sont très variées et n'ont en commun que ... cette contrainte d'équivalence, comme le montre ce petit échantillon :

/A/ Il y a beaucoup d'imbéciles au sein de l'Université $\leftrightarrow$ L'Université est peuplée d'imbéciles

/B/ Tu as de beaux yeux $\leftrightarrow$ Tes yeux sont beaux

/C/ Je l'aime à la folie $\leftrightarrow$ J'en suis follement amoureux

/D/ Mathilde ne viendra pas $\leftrightarrow$ Quelqu'un dit d'une personne qui s'appelle Mathilde qu'elle ne se déplacera pas pour aller à l'endroit où il se trouve.

En /A/ la relation entre Il y a beaucoup de $N$ au sein de Det $N$ et Det $N$ est peuplée de $N$ est à la fois structurelle et lexico-sémantique. Elle ne se vérifierait plus si on remplaçait beaucoup de $N$ par peu de $N$ ou un certain nombre de $N$. L'équivalence y est le résultat d'une restructuration, c'est-à-dire d'un changement, lexicalement dépendant, de structure.

La restructuration en /B/ entre Pro avoir Det Adj $N$ et $A d j$-pos $N$ être Adj diffère de celle que l'on a en /A/. Elle dépend de la grammaire des deux principaux auxiliaires du français et a, de ce fait, un domaine d'application beaucoup plus général : Ils ont des pantoufles confortables $\leftrightarrow$ Leurs pantoufles sont confortables; Cette robe a une coupe originale $\leftrightarrow$ La coupe de cette robe est originale ${ }^{10}$.

En /C/, l'équivalence est le produit d'une translation entre les catégories du nom et de l'adverbe.

Enfin, en /D/, le deuxième terme de l'équivalence est une description définitoire de type métalinguistique du premier.

Comme l'a démontré Lavoisier, les changements dans l'équivalence permettent, mieux que n'importe quelle autre expérience, de connaître précisément la nature, la constitution et l'analyse de ce qui fait l'objet de l'expérience. Il n'est donc pas exagéré d'affirmer que l'analyse matricielle définitoire qui ne se déploie que sous la contrainte de l'équivalence est a priori un bon outil d'exploration de la constitution des énoncés.

$\overline{10}$ Où l'on constate que le passage d'un pronom à un déterminant suivi d'un nom, d'un côté, et d'un adjectif possessif à un groupe nominal formé d'une suite $\operatorname{Det} N$ de $\operatorname{Det} N$, de l'autre, ne modifie en rien la relation d'équivalence. 
Si l'on admet que les formes les moins contraintes sont aussi les formes les plus simples, on peut poser d'emblée que la matrice d'un énoncé est sa forme la plus simple et qu'un énoncé est d'autant plus complexe qu'il y a d'étapes pour le constituer à partir de sa matrice.

Mais s'il est clair qu'une matrice est plus simple que l'énoncé le plus synthétique qui en est issu, peut-on mesurer des degrés de complexité d'énoncés issus d'une même matrice ou de matrices également simples? Et doit-on considérer que des énoncés apparemment très simples et qui sont justiciables d'une analyse matricielle très complexe sont en fait des énoncés complexes? On ne peut répondre qu'en se penchant sur les origines de la complexité.

\section{5. À l'origine de la complexité : la triple adéquation}

La musique a ceci en commun avec la langue que l'articulation de deux ou trois constituants élémentaires, dès lors que leur accord est validé par le plaisir qu'on en a retiré, suggérera toujours un sens à autrui. La langue ajoute à la musique des mots qui ont la réputation d'être plus étroitement chevillés surtout lorsqu'ils se moulent dans les formes, les combinaisons et l'ordre d'une grammaire et que leur assemblage sonne bien et produit un sentiment de complétude qui rappelle et produit le même effet qu'une mélodie dont les contours viennent de s'achever.

De la syllabe à l'énoncé, une forme ou une autre d'accord harmonique, commande directement les différentes étapes du développement d'une langue. C'est d'abord l'infrastructure harmonique - distribution réglée des consonnes et des voyelles, accentuation, variation de la quantité et des fréquences - des premières syllabes ou des premiers mots émergents qui détermine, à l'origine, une première adéquation à des représentations encore abstraites et paradigmatiques du sens. C'est ensuite une réanalyse des constituants sonores élémentaires et indécomposables de ces entités syllabiques qui amène l'être humain à prendre conscience de ce que les linguistiques ont fini par appeler phonèmes et à les combiner de telle sorte qu'ils correspondent, dans ce que l'on 
pourrait appeler une deuxième adéquation, à des catégories de mots dotés de fonctions et désignant des référents concrets ou abstraits relativement précis. C'est enfin la troisième adéquation, configuration linéaire de la combinaison des mots en fonction de contraintes prosodiques indissociables de la production du sens, qui parachèvera la complétude harmonique et mélodique de tout l'énoncé et permettra la perception d'une prédication ${ }^{11}$.

5.1. Adéquation harmonique élémentaire et complexité concaténative

Les premiers homo sapiens, comme aujourd'hui les tout petits, se sont d'abord adonnés à des lallations, à un babillage, un gazouillis, une scansion, un fredonnement, un chantonnement dont la modulation, encadrée, encouragée et limitée par les possibilités de leur appareil phonatoire, leur a permis, de manière holistique, de se familiariser avec une gamme de syllabes possibles et un éventail de possibilités d'accentuation et d'enchaînement. Le parachèvement de cette activité d'articulation a coïncidé lorsqu'il atteignait son but à un "sentiment de complétude ». C'est, en effet, par les différents aspects de leur versant sonore que les langues, à leur naissance ou à leur renaissance dans la bouche des enfants, ont accédé au sens.

Les hommes, les premiers, comme les enfants, comme nous, découvrent vite que la structuration des formes, surtout quand elles sont sonores, leur raréfaction et leur remodelage, l'organisation de plus en plus contrainte de leur durée et de l'éventail de leurs modulations, leur segmentation, l'économie de leur redon-

11 Nous renvoyons ici à notre développement (Amr Helmy Ibrahim, " Les conditions de la prédication dans les langues ", Prédicats, prédication et structures prédicatives, Paris, CRL, 2009, p. 12-49 et "Supports d'actualisation et dualité constitutive de la prédication ", Supports et prédicats non verbaux dans les langues $d u$ monde, Paris, CRL, 2010, p. 36-73) du mécanisme, l'un des plus sûrement distinctifs et définitoires des langues naturelles et de l'espèce humaine, baptisé presque simultanément en France (André Martinet, Éléments de linguistique générale, Paris, Armand Colin, 2003 [1960]) [Pour la double articulation, cf. p. 13-15] et aux États-Unis (Charles F. Hockett, "The Origin of Speech ", Scientific American, 203/3, 1960, p. 89-97) " double articulation" ou duality of patterning. 
dance, leur permettent de simuler ce qu'ils veulent exprimer. La galaxie sonore offre à l'homme des différentiels qui lui permettent d'anticiper les principales formes grammaticales : foyer prédicatif, prédications secondes, actants ou arguments, marqueurs de temps, de quantité, de mouvement, de direction, de manière.

Le moule sonore anticipe pratiquement toute la grammaire. Cette première adéquation opère entre une forme sonore et ce que l'on pourrait appeler la forme du sens, l'infrastructure formelle de la signification. Mais il n'y a pas encore à proprement parler de langue.

À ce premier stade, la complexité vient de l'obligation de traiter simultanément tous les constituants de l'infrastructure prosodique en étant tendu vers la construction d'un sens dont on n'a qu'une représentation abstraite. L'adéquation de cette infrastructure prosodique primitive à une représentation très schématique, qui va servir de paradigme sémantique de référence, enclenche la dynamique de construction de la grammaire de la langue. Elle anticipe par l'entremise des traits prosodiques ce que seront les contraintes syntaxiques et fonctionnelles de la grammaire. Les modulations prosodiques enchaînent les sons en opérant une première segmentation selon des schémas harmoniques qui sont d'abord essentiellement syllabiques, puis propositionnels et enfin phrastiques. Cette modulation segmentée constitue un moule où le foyer prédicatif trouvera une place aux contours sonores distincts. Cette première adéquation assigne également leurs places aux principaux arguments du prédicat, parfois à une prédication seconde accompagnée elle aussi de ses arguments, et anticipe les conditions de complétude de l'énoncé. Le placement des différents types d'accents joue un rôle central dans ce processus. La complexité prend donc ici sa source dans trois dimensions : premièrement, le fait que le locuteur traite simultanément les mécanismes de l'intonation, de l'accentuation, du découpage rythmique, de l'articulation et de l'assignation des fréquences; deuxièmement, le fait que ce traitement conduit à la production d'unités de tailles variables qui doivent être recyclées en production comme en reconnaissance au fur et à mesure de 
l'avancement du processus; troisièmement, le fait que le résultat du processus doit correspondre à une adéquation avec un modèle potentiellement paradigmatique de référence, c'est-à-dire une représentation abstraite, perçue comme récurrente et qui fait sens.

Cette première forme de complexité pourrait être illustrée, dans sa forme achevée, par tous les effets que peut produire un fredonnement juste, c'est-à-dire par le pouvoir qu'a le fredonnement correct d'une ligne mélodique d'évoquer une production musicale dans sa totalité, sa richesse et sa complexité. Elle est donc, d'une certaine manière, de nature concaténative, mais elle est aussi totalement grammaticalisée au sens où ses constituants ne sont pas complexes en eux-mêmes mais par la complexité qu'ils ont de pouvoir évoquer et par celle qui caractérise le parcours au terme duquel ils ont été produits. Elle n'est pas mesurable du fait que ses mécanismes et les relations qu'ils impliquent sont isomorphes et se manifestent au même degré dans toutes les langues. Le parcours suivi par la première adéquation n'en est pas moins à l'origine de la différenciation des langues.

5.2. Adéquation catégorielle, deuxième adéquation et première forme de complexité intégrative

Ce n'est que lorsque l'entrecroisement, le recoupement, les remises en ordre partielles de ces mouvements mélodiques seront devenues assez familières et assez largement partagées que des séries de réanalyses dégageront plus précisément une liste fermée de phonèmes, une liste fermée de syllabes, un protocole de construction des mots et surtout un petit nombre de catégories et que le champ sera ouvert à une combinatoire régie par le besoin d'attribuer $\mathrm{x}$ à $\mathrm{y}$, de mettre $\mathrm{x}$ et $\mathrm{y}$ en relation et d'étalonner le mouvement et le changement. Le locuteur nomme, désigne, étiquette, labellise. Cette production lexicale de plus en plus intense, qui s'accompagne de la mise en relation de deux ou trois mots, construit alors ses déclinaisons, ses flexions, ses schèmes de dérivation. 
Ce deuxième temps est mû par un besoin d'adéquation catégorielle. Les mots créés, repris ou découverts n'entrent alors dans la danse, ne peuvent trouver leur place dans le flux mélodique qu'à condition d'appartenir à une catégorie et de se soumettre à son protocole de fonctionnement. L'adéquation catégorielle est régie essentiellement par des mécanismes applicatifs - relations opérateur / opérande ou opérateur / arguments, notamment. On assiste alors à une extension rapide de l'étiquetage, de l'attribution de $\mathrm{x}$ à $\mathrm{y}$, de la mise en relation de $\mathrm{x}$ avec $\mathrm{y}$ et de la standardisation, on peut aussi dire de la normalisation de ces attributions et de ces mises en relation.

La complexité de cette deuxième adéquation est différente de la première : c'est la première étape de la complexité intégrative. Elle se caractérise, elle aussi, par la gestion simultanée de paramètres multiples et hétérogènes : mise en place d'indicateurs relationnels des accords de nombre, de genre, de personne qui assurent les liens entre les mots; application des opérateurs de perfectivation et d'imperfectivation; opérations de détermination et de quantification; application des opérateurs de phase ${ }^{12}$ et des opérateurs de succession temporelle. Elle est très précisément

12 Il est aujourd'hui généralement admis que dans toutes les langues du monde, les procès, quelle que soit la catégorie grammaticale dans laquelle ils s'expriment - verbe, nom, adverbe... - peuvent être repérés ou situés, mais aussi déterminés par rapport à trois paramètres distincts : le moment du déroulement du procès (c'est ce qu'on appelle en français " temps " - on dit aussi pour éviter les confusions " temps grammatical ", en anglais tense, en allemand tempus / tempora), la phase du déroulement du procès et la manière avec laquelle il se déroule (les deux derniers paramètres sont souvent regroupés en français et en anglais sous la dénomination aspect / aspect, en allemand, la phase correspond à Aspekt et la manière à Aktionsart). La phase du procès a en commun avec le moment du procès qu'elle correspond à une saisie et non à la nature même du procès. On choisit de saisir le procès à son commencement, à sa fin, dans sa totalité, dans son déroulement, de même qu'on choisit de situer le procès dans un moment antérieur, contemporain ou postérieur au moment où l'on parle. La nature lexicale du procès est un produit de sa perfectivation ou de son imperfectivation ainsi que de la manière avec laquelle il se déroule, c'est-à-dire de son mode d'action - son Aktionsart (voir Amr Helmy Ibrahim, "Moment, phase et manière du procès dans les matrices analytiques définitoires ", Philologica, Zbornik Filozofickej Fakulty Univerzity Komenského (Recueil de travaux de la Faculté des Lettres de l'Université Comenius), Bratislava, 2008, p. 83-93). 
mesurable au cours de l'analyse matricielle. Comme on l'a évoqué dans l'étalonnage de la simplicité (au point 2) cette mesure sera le produit d'une double procédure :

/1/ La détermination du nombre et de la nature des opérations et des étapes qui mènent de la matrice complète aux formes observées.

/2/ L'évaluation de l'accessibilité de la forme observée, c'està-dire la détermination du fait qu'elle constitue ou non un dénominateur commun à un éventail maximal d'usages qu'ils soient simples ou complexes, grammaticalisés ou non. La pondération de l'importance des deux volets de la procédure produit la mesure finale, mais cette pondération n'a de sens qu'au terme de la troisième adéquation.

5.3. Troisième adéquation mélodique et signifiante : achèvement de la grammaticalisation et de la complétude des schémas de phrases

Il n'y a pas d'énoncé achevé sans un étalonnage du changement et du mouvement, sans la distinction parmi les changements possibles de ceux qui ne changent pas le sens, ceux qui le font plus ou moins varier et ceux qui le modifient radicalement. C'est à ce stade que se spécifient, à travers la construction de classes d'équivalence, les outils du fonctionnement grammatical - translations ${ }^{13}$, transformations et restructurations - et que les mécanismes de dérivation normalisent leurs schémas et leurs protocoles.

Au cours de ce troisième temps, le locuteur s'oriente vers une adéquation mélodique et signifiante où il retrouve, enrichi par l'acquis de l'adéquation catégorielle, l'adéquation harmonique élémentaire.

L'exploration des conditions et des types d'équivalence entre les énoncés dans le but d'étalonner la fonction du changement dans la construction du sens suit nécessairement quatre voies plus ou moins interdépendantes : (1) la pratique des "translations", (2) la pratique des " transformations " : changements opérant

13 Changement, au cours d'une restructuration, de la catégorie grammaticale d'une entrée lexicale, sans changement de sens de l'énoncé. 
automatiquement entre deux index structuraux, (3) la pratique des "restructurations " : changement de structure dépendant de la nature lexicale de ses constituants et, enfin, (4) la pratique des " paraphrases sémantiques " : changement de configuration des constituants de l'énoncé en fonction de leurs champs lexicaux et sémantiques sans que l'équivalence obtenue ne soit le produit d'une quelconque régularité structurale ou lexicale, ni de contraintes spécifiques d'appropriation.

L'exploration des équivalences, selon les trois premières voies et plus particulièrement dans la pratique des translations et des restructurations, génère des descripteurs spécifiques - notamment des supports, des classifieurs et des corrélats ${ }^{14}$ - et induit l'application systématique d'opérateurs d'intonation et d'opérateurs métaphrastiques en plus des opérateurs constitutifs de la deuxième adéquation.

Plus généralement, l'exploration des possibilités d'équivalence entre énoncés selon les quatre voies conduit à deux formes d'extension du sens : une extension centrifuge et une extension centripète. La première produit de la polyvalence et de la polysémie, la seconde construit des familles d'énoncés dont l'équivalence est le produit soit de dérivations simples soit de distributions complémentaires.

\subsubsection{Polyvalence et extension centrifuge des classes d'équivalence de l'adéquation mélodique et signifiante}

Lorsqu'une forme est polyvalente, polysémique ou polyfonctionnelle, chacune de ses valeurs entre dans une classe d'équivalence spécifique. La sélection de la valeur adaptée à un contexte donné exige alors la connaissance des différences existant entre ces classes spécifiques. La nature de cette équivalence

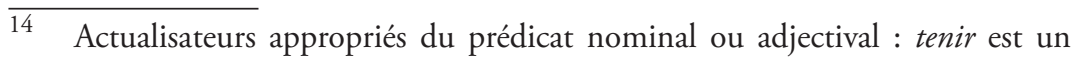
verbe support dans tenir une réunion; couleur est un nom classifieur dans de couleur rouge; taille est un corrélat dans de grande taille. 
- transformation ${ }^{15}$, restructuration ${ }^{16}$ ou paraphrase hapax ${ }^{17}-$ et le nombre d'équivalences possibles pour une même forme constituent des critères mesurables de complexité. Si, par exemple, je prends le mot bien, il correspond, entre autres, à :

(1) Une bonne chose dans :

(a) C'est bien! $\leftrightarrow$ (a') C'est une bonne chose!

Tu as bien fait de venir $\leftrightarrow$ C'est une bonne chose que tu sois venu $\leftrightarrow$ Ta venue est une bonne chose.

(2) (En (bonne) forme + De bonne bumeur + À l'aise) dans :

(a) Je me sens bien $\leftrightarrow$ (a') Je me sens (en (bonne) forme + de bonne humeur + à l'aise)

(3) De bonnes actions dans :

(a) Elle fait le bien $\leftrightarrow$ (a') Elle fait de bonnes actions.

(4) (De qualité + Respectable) dans :

(a) C'est (une personne + quelqu'un de) bien $\leftrightarrow$ (a') C'est (une personne + quelqu'un de) (qualité + respectable $) \leftrightarrow$ C'est (une personne + quelqu'un) que (je + on) juge (de qualité + respectable).

(5) De manière satisfaisante dans :

(a) Il travaille bien $\leftrightarrow$ (a') Il travaille de manière satisfaisante $\leftrightarrow$ Il travaille d'une manière jugée satisfaisante.

15 Changement de construction, dépendant essentiellement de la catégorie et de l'ordre des constituants de cette structure mais non, ou très peu, de leur nature lexicale, comme peuvent l'être la passivation, l'extraction, la transformation relative, la transformation impersonnelle, l'extraposition, la transformation de longueur, la transformation de montée du sujet, etc.

Changement de structure dépendant de la nature lexicale du constituant. Ainsi (a) J'aime Lou qui a la structure $P R O^{\circ}$-hum $V N 1$-hum peut être restructuré en (a') J'éprouve de l'amour pour Lou qui a la structure $P R O^{\circ}$-hum $V$-sup Prep-de $N$-v Prep-pour $N$-hum, mais (b) Je connais Lou qui a la même structure que (a) ne peut pas être restructuré en (b') *J'éprouve de la connaissance pour Lou y compris en substituant au verbe support éprouver un autre verbe support. La nominalisation des verbes aimer ou éprouver ne constitue donc pas une transformation mais une restructuration.

17 C'est-à-dire une paraphrase isolée qui n'est possible sous cette forme qu'avec cette entrée lexicale. 
(6) (La mention + L'appréciation) bien dans :

(a) Nous vous avons mis un "Bien" $\leftrightarrow$ (a') Nous vous avons accordé (la mention + l'appréciation) "bien".

(7) (Bon! + D'accord) dans :

(a) Bien! Bien! On fera comme tu veux $\leftrightarrow$ (a') (Bon! Bon! + D'accord! D'accord!) On fera comme tu veux.

(8) Très dans :

(a) Je vous trouve bien pâle! $\leftrightarrow$ (a') Je vous trouve très pâle!

(9) Assurément et cela me surprend dans :

(a) C'est bien la première fois que je vois ça $\leftrightarrow$ (a') C'est assurément la première fois que je vois cela et cela me surprend.

(10) X et pas un autre dans :

(a) C'est bien lui. $\leftrightarrow$ (a') C'est lui et pas un autre.

(11) Tout à fait dans :

(a) Ça te ressemble bien! $\leftrightarrow$ (a') C'est tout à fait toi $\leftrightarrow$ Cela (correspond + ressemble) tout à fait à ce que (tu es + l'on sait de toi).

(12) En (accord + harmonie) dans :

(a) Cette robe te va bien. $\leftrightarrow$ (a') Cette robe est en (accord + harmonie) avec ce que tu es.

(13) Inverseur de valeur dans l'intensification dans :

(a) C'est bien beau! $\leftrightarrow$ (a') C'est très (mal + moche)!!

(14) Je l'aime mais pas d'amour dans :

(a) Je l'aime bien $\leftrightarrow$ (a') Je l'aime mais pas d'amour.

(15) Volontiers dans:

(a) J'en reprendrais bien (un morceau) $\leftrightarrow$ (a') J'en reprendrais volontiers (un morceau).

Je veux bien en reprendre (un morceau) $\leftrightarrow$ J'en reprendrais

18 (13a), nous fait-on remarquer, "suppose au contraire de toutes les autres relations une forme d'antiphrase, quelque chose qui relève de l'ironie et ne peut absolument pas s'intégrer à ce niveau " du fait qu'il appartient à un registre rhétorique. Effectivement. Il n'en reste pas moins, malgré cette hétérogénéité des niveaux d'analyse qu'il existe une équivalence structurelle entre (13a) et sa paraphrase en (13a') (voir plus bas 13'). 
volontiers (un morceau) $\leftrightarrow$ *? Je (veux + voudrais) volontiers en reprendre (un morceau).

Je vous ressers? - Volontiers! $\leftrightarrow$ Je veux bien!

(16) Structurellement polysémique dans :

/ $\alpha /$ Vous êtes bien sur Radio Classique $\leftrightarrow / \alpha-1 /$ (Vous vous sentez bien sur Radio Classique + / $\alpha-2 /$ Vous ne vous êtes pas trompé, vous êtes effectivement sur Radio Classique) ${ }^{19}$.

$/ \beta$ / Il a bien démonté le chauffe-eau ${ }^{20} \leftrightarrow$

( $\beta$ 1) Il a démonté le chauffe-eau d'une manière satisfaisante.

( $\beta$ 2) C'est (bien) ce qu'il a fait. Il a démonté le chauffe-eau, (n'est-ce pas?)

( $\beta$ 3) (Il prétend qu'il ne sait rien faire + Il a dit qu'il n'avait rien fait) mais il a (pourtant + quand même) démonté le chauffe-eau.

Sans entrer dans le détail des analyses, on remarque rapidement une corrélation relativement forte entre chacune des 19 valeurs différentes qu'a prises bien et, à l'exception de (16), à chaque fois une configuration structurelle, catégorielle et contextuelle spécifique. À chaque fois on peut dire qu'il existe une classe d'équivalence entre la configuration structurelle où apparaît bien et celle de l'énoncé qui la paraphrase :

(1') Prés bien! $\leftrightarrow P R O_{\text {-dem }} V$-être $\operatorname{Det}_{\text {-ind }} A d j_{\text {-bon }} N_{\text {-gén }}$

$P R O_{\text {-pers }} V_{\text {-faire }}$ bien de $V_{\text {-inf }}(\Omega) \leftrightarrow P R O_{\text {-dem }} V$-être $D e t_{\text {-ind }} A d j_{\text {-bon }}$ $N_{\text {-gén }}$ Que $P \leftrightarrow A d j_{\text {-pos }} N_{\text {-v }} V_{\text {-être }} \operatorname{Det}_{\text {-ind }} A d j_{\text {-bon }} N_{\text {-gén }}$

(2') $P R O_{\text {-pers }}$ se $V_{\text {-sentir }}$ bien $\leftrightarrow P R O_{\text {-pers }}$ se $V_{\text {-sentir }}$ Prep (Prep

19 Il n'y a pas de différence d'intonation, d'accentuation ou de schéma prosodique entre les deux valeurs. Cette ambiguïté structurelle parfaite fait de cet énoncé, que l'on peut effectivement entendre (2009-2010) sur cette radio française, une trouvaille publicitaire remarquable.

20 L'interprétation correspondant à $(\beta 1)$ est prosodiquement distincte des interprétations $(\beta 2)$ et $(\beta 3)$ qui, elles, ne semblent pas pouvoir être départagées prosodiquement. Certaines structures prosodiques peuvent entraîner des interprétations qui n'ont pas été envisagées ici. C'est le cas notamment d'une manière de produire C'est très bien...ça remarquablement bien analysée par Nathalie Sarraute dans Pour un oui ou pour un non, Paris, Édition de Poche, Folio Théâtre, 1999, [1982], p. 26-28. 
$\left(A d j_{\text {-bonne }}\right)$ forme $+\operatorname{Prep}_{-d e} A d j_{\text {-bonne }}$ bumeur + Prep $_{-\dot{a}}$ l'aise $)$

(3') $P R O_{\text {-pers }} V_{\text {-faire }} D e t_{\text {-le }}$ bien $\leftrightarrow P R O_{\text {-pers }} V_{\text {-faire }} \operatorname{Det} A d j_{\text {-bon }}$ action(s)

(4') $P R O_{\text {-dem }} V_{\text {-être }}\left(\right.$ Det $_{\text {-un(e) }} N_{\text {-génér }}+P R O_{\text {-indéf }}$ Prép $\left._{\text {-de }}\right)$ bien $\leftrightarrow$ $P R O_{\text {-dem }} V_{\text {-tetre }}\left(\right.$ Det $_{\text {-un(e) }} N_{\text {-génér }}+P R O_{\text {-indéf }} P$ rép -de $)($ qualité + respectable $)$

(5') $P R O^{\circ}{ }_{\text {-pers }} V_{\text {-travailler }}$ bien $\leftrightarrow P R O^{\circ}{ }_{\text {-pers }} V_{\text {-travailler }}$ de manière $A d j_{\text {-satisfaisante }} \stackrel{\text {-pers }}{\leftrightarrow} P R O^{\circ}{ }_{\text {-pers }}^{\text {-travailer }} V_{\text {-travailler }}$ de manière jugée $A d j_{\text {-satisfaisante }}$

(6') $P R O_{\text {-pers }} P R O_{\text {-pers }} V_{\text {-mettre }}$ (Det-un) "bien » $\leftrightarrow P R O_{\text {-pers }}$ $P R O_{\text {-pers }} V_{\text {-mettre }} \operatorname{Det}($ mention + appréciation) "bien »

(7’) Bien! Bie! P $\leftrightarrow$ (Bon! Bon! + D'accord! D'accord!) P

(8') $P R O_{\text {-pers }} P R O_{\text {-pers }} V_{\text {-trouve }}$ bien $A d j_{\text {-gradable }} \leftrightarrow P R O_{\text {-pers }} P R O_{\text {-pers }}$ $V_{\text {-trouve }}$ très $A d j_{\text {-gradable }}$

(9') Prés bien Det $t_{\text {-def }}$ première fois Que $P \leftrightarrow P R O_{\text {-dem }} V_{\text {-être }}$

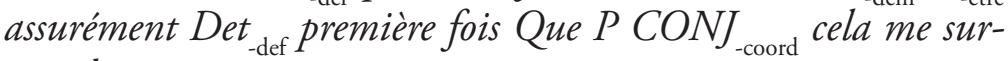
prend.

(10') Prés bien $P R O_{\text {-pers }} \leftrightarrow P R O_{\text {-dem }} V_{\text {-être }} P R O_{\text {-pers }} C O N J_{\text {-coord }}$ $\mathrm{Neg}\left(\mathrm{Det}_{\text {-ind }} P R O_{\text {-ind }}+P R O_{\text {-ind }} \operatorname{Prep} \mathrm{Adj}_{\text {-autre }}\right)$

(11') $P R O_{\text {-dem }} P R O_{\text {-pers }} V_{\text {-ressembler }}$ bien $! \leftrightarrow P R O_{\text {-dem }} V_{\text {-être }}$ tout à fait $P R O_{\text {-pers }}^{\text {-dem }} \leftrightarrow P R O_{\text {-dem }}\left(V_{\text {-correspondre }}+V_{\text {-ressembler }}\right)^{\text {-pers }}$ tout à fait Prep $_{\text {-à }} P R O_{\text {-dem }} Q U E\left(P R O_{\text {-pers }} V_{\text {-être }}+P R O_{\text {-pers }}^{\text {Prep } P R O)}\right.$

(12') $A d j_{\text {-dem }} N_{\text {-parure }} P R O \mathrm{i}_{\text {-pers }} V_{\text {-aller }}$ bien $\leftrightarrow A d j_{\text {-dem }} N_{\text {-parure }} V_{\text {-être }}$ Prep $_{\text {-en }}$ (accord + harmonie) Prep -avec $_{\text {- }} P R O_{\text {-dem }}$ Que PROi ${ }_{\text {-pers }} V_{\text {-être }}$ (13’) Prés bien $A d j \leftrightarrow P R O_{\text {-dem }} V_{\text {-être }}$ très $A d j j_{\text {-neg }}$

(14') $P R O_{\text {-pers }}^{\circ} P R O 1_{\text {-pers }} V_{\text {-aimer }}$ bien $\leftrightarrow P R O_{\text {-pers }} P R O_{\text {-pers }} V_{\text {-aimer }}$ mais $N e g N_{-\mathrm{v}}^{-\mathrm{pess}}$

(15') $P R O_{\text {-pers }}^{\circ} P R O 1_{\text {-nr }} V_{\text {- }}$ vouloir $\operatorname{bien}(\Omega) \leftrightarrow P R O_{\text {-pers }}^{\circ} P R O 1_{\text {-nr }}$ $V_{\text {- } \neq \text { vouloir volontiers }}(\Omega)$

$P R O^{\circ} V_{\text {-pers }} V_{\text {-vouloir }}$ bien $P R O 1_{\text {-nr }} V_{\mathbf{~}_{\text {-inf } \neq \text { vouloir }}(\Omega) \leftrightarrow P R O^{\circ}}{ }_{\text {-pers }}$ $P R O 1_{\text {-nr }}^{\text {-pers }} V_{\text {-futur }}^{\text {-vouloir vouloir }}$ volontiers $(\Omega) / /$ Volontiers $\leftrightarrow P R O_{\text {-pers }}^{\text {-pers }}$ $V_{\text {-vouloir }}$ bien 
(16') $/ \alpha / P R O_{\text {-pers }}^{\circ} V_{\text {-etre }}$ bien Prep -sur $_{\text {-nr }} \leftrightarrow\left(P R O_{\text {-pers }} P R O_{\text {-pers }}\right.$ $V_{\text {-sentir }}$ bien Prepp pers $^{- \text {-etre }} N_{\text {-ni }}+$ Vous ne vous êtes pas trompé $P R O_{\text {-pers }}^{\circ} V_{\text {-être }}$ effectivement Prep -sur $_{\text {- }} N$-nr

$/ \beta / P R O^{\circ}{ }_{\text {-pers }} V$ bien $\operatorname{Det} N_{- \text {-nr }} \leftrightarrow$

$(\beta 1) P R O_{\text {-pers }}^{\circ} V$ Det $N_{- \text {-n }}$ de manière satisfaisante

( $\beta$ 2) Prés (bien) $P R O_{-\mathrm{nr}}$ Que $P R O_{\text {-pers }}^{\circ} V_{\text {-proforme faire }}$ (n'est-ce pas)?

(B 3) $P$ mais $P R O_{\text {-pers }}^{\circ} V$ (pourtant + quand même) Det $N_{\text {-nr }}$

Ce sont, en effet, à chaque fois des paradigmes et une construction entière qui sont concernés par l'équivalence et non seulement des termes isolés, et ce même quand l'équivalence constatée est unique ou ne porte que sur un nombre très faible d'entrées lexicales. Le degré de complexité sous-jacent à la valeur choisie pourrait être évalué à partir de trois repères : /1/ une distance sémantique relative par rapport à une valeur présumée "de base ", par exemple celle de bon; /2/ le nombre et la nature des modifications que la paraphrase de la construction où se trouve bien lui apporte ainsi que le degré de complexité de cette construction elle-même; /3/ le fait que la même forme puisse entrer dans plus d'une classe d'équivalence comme en (16'). Mais ce raisonnement de "bon sens " se heurte à trois difficultés. Il est très difficile pour ne pas dire impossible d'établir une valeur de base, un signifié de départ de bon, voire, comme on le fait dans certaines approches sémantiques, de tenter de trouver un dénominateur commun aux usages de bon à partir duquel on décrirait tous ses usages. Et même à supposer que cette entreprise ait un sens et qu'elle soit matériellement possible, il faudrait un ouvrage pour chaque entrée lexicale ${ }^{21}$ et les résultats, malgré l'intelligence avec laquelle ils auraient été obtenus, seraient à la fois invérifiables et impossibles à généraliser. Par contre, et à condition de disposer d'une mesure du coût et des effets des modifications qui affectent une structure, on devrait pouvoir comparer des différences de constructions liées à des différences d'interprétation sémantique et de contextualisation pragmatique

$21 \quad$ Voir Daniel Duprey, L'Universalité de 'bien', Bern, Peter Lang, 1995, 200 p. 
et en déduire des degrés de complexité. On ne compare pas dans ce cas des constructions syntaxiques dans l'absolu, mais on établit les étapes par lesquelles se complexifie la relation entre un signifiant et les formes morphosyntaxiques qui sont associées à son interprétation sémantique. Ce faisant, on se heurte cependant à la deuxième difficulté : en observant les classes d'équivalence des index structuraux correspondant aux différentes valeurs que prend le signifiant bien, on constate, d'une part, que les degrés de complexité des constructions, quelles qu'elles soient, qui contiennent bien et les constructions qui les paraphrasent sont de nature hétérogène et, d'autre part, que la sélection des constituants à l'intérieur de chacun des paradigmes des deux types de constructions ne semble pas obéir à une même logique. En bref, un lexème peut se retrouver dans un paradigme du fait des propriétés générales ou catégorielles de ce paradigme, mais il peut aussi s'y retrouver parce qu'il est dans une relation d'appropriation avec le constituant du paradigme précédent ou suivant, autrement dit qu'on n'a pas une articulation régulière des contraintes paradigmatiques aux contraintes syntagmatiques et qu'il est par conséquent difficile de rapporter la mesure de la complexité au calcul de l'articulation de ces contraintes. Enfin, troisième difficulté, il existe des cas comme (16) où la construction où se trouve bien peut entrer dans plus d'une classe d'équivalence et que, d'une part, il n'y pas de marque formelle dans la construction qui permette de détecter la présence d'une ambiguïté structurelle et, d'autre part, on ne voit pas sur quel critère on peut se fonder pour sélectionner l'une des valeurs possibles de (16' $\alpha)$ ou (16' $\beta)$.

Faut-il pour autant, malgré l'existence de ces relations extrêmement précises et très précisément réglées dans le cadre de classes d'équivalences entre les énoncés qui contiennent bien et les énoncés restructurés qui les reformulent en les expliquant et en levant toute ambiguïté sur leur sens, renoncer à mesurer une complexité dont nous avons une perception intuitive indiscutable? Certainement pas. Observons le tableau suivant. 


\section{Tableau 1}

\begin{tabular}{|c|c|c|c|c|c|c|}
\hline & Énoncé avec bien & $\begin{array}{l}\text { Sup }_{\text {-occur }}{ }^{i} \\
+{ }_{\text {+Préd_-géni }} \\
\text { + Préd-red }_{\text {iii }} \\
+ \text { paeisaa }^{\text {iv }}\end{array}$ & $\begin{array}{l}\text { Det / Corrélat } \\
\mathbf{N}_{\text {-clas }} \text { / } / N_{\text {-gén }}\end{array}$ & Prédicat & $\begin{array}{l}\mathbf{N}_{\text {-gén }} / \mathbf{N}_{\text {-clas }} \\
\mathbf{N}_{\text {-op }}^{\text {vii }}\end{array}$ & Extension \\
\hline \multirow[t]{2}{*}{1} & Prés bien & Prés & Det & bonne & Chose & $\varnothing$ \\
\hline & Pro $\mathrm{V}$ bien de $\mathrm{V}_{\text {-inf }}$ & Prés & Det & bonne & Chose & Que P \\
\hline 2 & Pro se $\mathrm{V}_{\text {-sentir }}$ bien & Pro seV & $\begin{array}{l}\text { Prep } \\
\text { Prep Det }\end{array}$ & $\begin{array}{r}\text { bonne } \\
\varnothing\end{array}$ & $\begin{array}{l}\text { forme }+ \\
\text { humeur }\end{array}$ & $\varnothing$ \\
\hline 3 & Pro $V_{\text {-faire }}$ Det bien & Pro $V_{\text {-faire }}$ & Det & bonnes & Actions & $\varnothing$ \\
\hline 4 & $\begin{array}{l}\text { Prés (Pro-indef } \\
\left.\text { de+Det } N_{\text {-gén }}\right)\end{array}$ & $\begin{array}{l}\text { Prés (Pro-indef } \\
\text { + Det } \mathrm{N}_{\text {-gén }} \text { ) }\end{array}$ & $\begin{array}{l}\text { qui a un }(\mathrm{e}) \\
(\text { statut }+ \\
\text { situation }+ \\
\text { origine) [jugée] } \\
\left(\text { Prep-de }_{-}+\mathrm{E}\right)\end{array}$ & $\begin{array}{l}\text { qualité } \\
\text { respectable }\end{array}$ & $\varnothing$ & $\varnothing$ \\
\hline 5 & Pro $\mathrm{V}_{\text {-travailler }}$ bien & Pro $V_{\text {-travailler }}$ & de manière & satisfaisante & $\varnothing \quad \varnothing$ & $\varnothing$ \\
\hline 6 & $\begin{array}{l}\text { Pro-pers } \text { Pro }_{\text {-pers }} \mathrm{V}_{\text {-mettre }} \\
\text { Det bien } \\
\end{array}$ & $\begin{array}{l}\text { Pro-pers } \\
\text { pers } V_{\text {-mettre }}\end{array}$ & Det & bien & $\begin{array}{l}\text { (mention+ } \\
\text { appréciation) }\end{array}$ & $\varnothing$ \\
\hline 7 & Bien! Bien! P & $\varnothing$ & $\varnothing$ & Bon! Bon! & $\varnothing$ & $P$ \\
\hline 8 & $\begin{array}{l}\text { Pro }_{\text {-pers }} \text { Pro }_{\text {pers }} \mathrm{V}_{-} \\
\text {trouver } \text { bien } \text { Adj-gradable }^{2}\end{array}$ & $\begin{array}{l}\text { Pro-pers } \\
\text { pers V-tro. } \\
\text {-trouver }\end{array}$ & $\varnothing$ & très & $\varnothing$ & Adj \\
\hline 9 & $\begin{array}{l}\text { Prés bien Det } \\
\text { première fois Que P }\end{array}$ & Prés & $\varnothing$ & assurément & $\begin{array}{l}\text { la première } \\
\text { fois }\end{array}$ & $\begin{array}{l}\text { Que P et cela } \\
\text { me surprend }\end{array}$ \\
\hline 10 & Prés bien $\mathrm{X}$ & Pred-red & et pas & bien & $\begin{array}{l}\text { (un autre }+ \\
\text { qq'un d'autre) }\end{array}$ & $\varnothing$ \\
\hline 11 & $\begin{array}{l}\text { Pro-dem } \text { Pro-pers }_{\text {V. }} \\
\text { ressembler bien }\end{array}$ & $\begin{array}{l}\text { Pred }_{\text {-red }} \\
\text { Pro-pers }\end{array}$ & $\varnothing$ & tout à fait & $\varnothing$ & $\varnothing$ \\
\hline 12 & $\begin{array}{l}\operatorname{Adj}_{\text {-dem }} \mathrm{N}_{\text {-parure }} \text { Pro } \mathrm{V}_{\text {. }} \\
\text { aller bien }\end{array}$ & $\varnothing$ & $\begin{array}{l}\text { Adj-dem }_{\text {-parure }} \mathrm{N}_{\text {-pre }} \\
\mathrm{V}_{\text {-être }}\end{array}$ & $\begin{array}{l}\text { En harmonie } \\
\text { avec }\end{array}$ & $\begin{array}{c}\text { ta } \\
\text { personne }\end{array}$ & $\varnothing$ \\
\hline 13 & Prés bien Adj! & Prés & $\varnothing$ & très & $\varnothing$ & Adj-neg $_{\text {-neg }}$ \\
\hline 14 & Pro $^{\circ}$ Pro $1 V_{\text {-aimer }}$ bien & $\varnothing$ & Pro $^{\circ}$ Pro 1 & $\mathrm{~V}_{\text {-aimer }}$ bien & $\varnothing$ & $\begin{array}{l}\text { mais sans } \\
\text { amour }\end{array}$ \\
\hline 15 & Pro $^{\circ} \mathrm{V}_{\text {-vouloir }}$ bien & $\varnothing$ & $\varnothing+$ Pro $^{\circ}$ & $\begin{array}{l}\text { Volontiers }+\mathrm{V} \text {. } \\
\text { vouloir bien }\end{array}$ & $\varnothing$ & $\varnothing$ \\
\hline \multirow[t]{2}{*}{$\begin{array}{c}16 \\
\mathrm{~A}\end{array}$} & \multirow[t]{2}{*}{$\begin{array}{l}\text { Pro } V_{\text {-être }} \text { bien Prep } \\
\mathrm{N}_{\text {-nr }}\end{array}$} & $\varnothing$ & \begin{tabular}{|l} 
Pro Pro V. \\
sentir
\end{tabular} & Bien & Prep N & $\varnothing$ \\
\hline & & $\begin{array}{l}\text { Préd-red }(\mathrm{Vs} \\
\text { ne vs êtes ps } \\
\text { trompés) }\end{array}$ & Pro $V_{\text {-être }}$ & Effectivement & Prep N & $\varnothing$ \\
\hline \multirow{3}{*}{$\begin{array}{c}17 \\
\beta\end{array}$} & \multirow{3}{*}{$\begin{array}{l}\text { Pro V bien Det N- } \\
\mathrm{nr}\end{array}$} & (1)Pro V & de manière & Satisfaisante & $\varnothing$ & $\varnothing$ \\
\hline & & (2)Pred-red & $\varnothing$ & Effectivement & $\varnothing$ & $\begin{array}{l}\text { ce que Pro V. } \\
\text { faire proforme }\end{array}$ \\
\hline & & $\begin{array}{l}\text { (3) } \mathrm{P}_{\text {-neg }} \\
\text { mais }\end{array}$ & $\varnothing$ & De fait & $\varnothing$ & $\begin{array}{l}\text { Pourtant } P+ \\
\text { qd même } P\end{array}$ \\
\hline
\end{tabular}

\footnotetext{
${ }^{i}$ Support d'occurrence : en français, les présentatifs c'est ou voilà, l'expression il y a. Pour une définition et une discussion voir Maurice Gross 1996 : 8-9, Amr Helmy Ibrahim, « Prolégomènes à une typologie de l'actualisation des noms ", dans Jacques François et Imtraud Behr (dir.), Les constituants prédicatifs et la diversité des langues, Mémoires de la Société de linguistique de Paris, Tome XIV, 2004, p. 64 et Amr Helmy Ibrahim, 2009, op. cit., p. 15-16.

ii Prédicat générique. C'est un prédicat à valeur très générale susceptible d'être un hypéronyme ou un classifieur du foyer prédicatif.

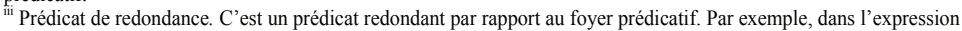
C'est une femme femme, la deuxième occurrence de femme est un prédicat de redondance. Voir Amr Helmy Ibrahim, L'analyse matricielle définitoire : un modèle pour la description et la comparaison des langues, À paraître.

${ }^{\text {iv }}$ Paeisaa : Proposition antérieure éventuellement implicite servant d'appui à l'assertion. C'est le cas notamment au bas du tableau de $17 \beta$ (3) où $I l$ a dit qu'il ne le réparerait pas mais - P-neg mais - constitue cette paeisaa.

" Les corrélats, Amr Helmy Ibrahim, 1998, p. 250, Amr Helmy Ibrahim, « Argumentation interne et enchaînements dans les matrices définitoires », dans Amr Helmy Ibrahim (dir.), Langages. Les discours intérieurs au lexique $\mathrm{n}^{\circ} 142,2001$, p. 106-112 et Amr Helmy Ibrahim, 2004, op. cit., p. 44), termes plus ou moins génériques, fournissent au prédicat non verbal des paramètres nécessaires à la construction de sa signification : état, forme, position, propriété, situation, statut, origine, obstacle, direction, manière, etc.). Ces paramètres ont un caractère large et moins approprié que les catégories étiquetées
} 
Si on part de la prédication minimale (1a) c'est bien, on constate que son sens se construit à travers une suite qui lui est équivalente (1a') c'est une bonne chose, formée de quatre constituants minimaux indécomposables : un support d'occurrence $\left(\right.$ Sup $\left._{\text {-occur }}\right)$ qui apparaît sous la forme du présentatif c'est, un corrélat $\left(C_{\text {-orrélat }}\right)$ qui prend la forme du déterminant une, le prédicat bonne et le nom générique chose. Les autres cas de figure, de (1) à (16), présentent des situations analogues à cette différence près que la relation entre l'énoncé avec bien et l'énoncé équivalent est plus complexe au sens où elle implique un plus grand nombre d'opérations et de changements pour parvenir à cette équivalence, qu'il s'agisse de choix aboutissant à une mise en parallèle ou à la reconstitution des constituants dont la réduction est nécessaire à l'interprétation.

L'énoncé (2) implique l'effacement d'un nom classifieur (forme, humeur, aise), (3) comporte une corrélation entre l'apparition du $V_{\text {-sup }}$ faire et du $N_{\text {-clas }}$ actions; (4) en plus d'introduire une double corrélation - entre un $N_{\text {-gén }}$ et un Corrélat et entre ce $C_{\text {-orrélat }}$ et le Prédicat - cela implique la réduction d'un jugement spécifique introduit par le $C_{\text {-orrélat }} ;(5)$ combine au prédicat satisfaisante un prédicat générique $V_{\text {-travailler }}$; on retrouve ce dédoublement prédicatif mais sous une autre forme en (6); (7) est le produit d'une opération plus simple mais d'une nature plus complexe eu égard aux conditions dans lesquelles elle se vérifie : la translation qui fait passer de bien à bon; l'interprétation de (9) ajoute à la spécificité du $N_{\text {-op }}$ la première fois qui la caractérise une extension qui n'est pas dérivée directement de la grammaire de bien; (10) et (11) introduisent une forme inédite de prédication dans le dédoublement prédicatif, celle qu'apporte le Pred ${ }_{\text {-red }}$ mais il n'a pas dans les deux cas la même portée : dans (11) il porte sur le pronom toi; à partir de (12) s'ajoutent à ces paramètres des enchaînements à chaque fois différents de dépendances lexicales, l'accumulation des paramètres engendrant des ambiguïtés structurelles voire, par exemple en (13) et en (16- $\beta 3$ ), des inversions d'orientation argumentative liées à un effet d'antiphrase ou à une extension externe en pourtant. 
Il est facile de retrouver ces situations d'équivalence dans n'importe quelle langue, que ce soit avec les mêmes cas de figure ou avec des cas de figure différents.

Phénomène essentiel à l'évaluation et à la mesure de la complexité : à l'exception du constituant en position de prédicat, les autres constituants, selon les langues ou les différents cas de figure qui apparaissent dans le tableau, peuvent apparaître ou être absents, être réduits ou effacés conformément à des contraintes explicites. Leur présence et leur position relèvent donc de choix de deux types :

/1/ Des choix concernant la manière avec laquelle chaque langue construit un sens particulier et qui porte sur le nombre d'opérations nécessaires pour que la formulation analytique dans une langue soit équivalente à la formulation synthétique correspondante. Ce dernier type de choix, à condition que la formulation analytique soit adéquate, permet d'identifier les spécificités du premier type de choix, c'est-à-dire de savoir quelles sont les opérations ou les constituants qu'une langue privilégie ou ignore et dans quel ordre de priorité.

12/ Des choix liés à la langue concernée - par exemple, en français on peut choisir de dire C'est bien! ou Bien! mais dans une langue comme l'arabe où il n'existe pas, dans ce contexte, d'équivalent au présentatif c'est il n'y a pas deux formes en concurrence et donc pas de choix; parallèlement, dans l'énoncé analytique le français interdit l'effacement du présentatif mais laisse le choix entre plusieurs de ses variantes (C'est + Cela est + Voilà $+\ldots$ ) une bonne chose, alors que l'arabe voit apparaître un présentatif de type déictique qui n’a pas de variantes.

Il devient ainsi possible de mesurer la complexité avec une relative simplicité et selon une double perspective sans jamais verser dans une forme ou une autre de glottocentrisme. D'une part, une mesure intrinsèque à une langue donnée qui compte les choix qui doivent être faits pour établir une équivalence stricte entre la formulation analytique et la formulation synthétique, d'autre part, une mesure relative aux choix, qu'on pourrait appeler adaptatifs, qu'impose au locuteur le passage à une langue 
- ou plus exactement la construction du sens dans une langue - à partir d'une autre langue, c'est-à-dire à partir des outils de construction du sens de cette autre langue. La complexité des choix adaptatifs est relative au locuteur et à sa langue maternelle.

L'unité de mesure associée au choix est de deux types : une substitution appropriée ou une réduction appropriée. Le deuxième type étant toujours plus coûteux que le premier. En effet, les substitutions appropriées sont données par le contexte et peuvent se faire même s'il n'y a pas eu une véritable appropriation du sens. Par contre, les réductions appropriées ne sont possibles que si le locuteur est capable de reconstruire le parcours de production du sens et de juger intuitivement de la qualité et du degré de précision de l'équivalence entre une formulation analytique et la formulation synthétique correspondante.

Dans le cas qui nous intéresse, la substitution appropriée concerne principalement le prédicat et est relativement régulière et homogène. Elle donne bonne dans (1), (2), (3) et (7), (qualité + respectable) dans (4), satisfaisante dans (5) et (16 $\beta-1)$, bien donc sans changement - dans (6), (10) et (16 $\alpha-1)$, bon dans (7), très $^{22}$ dans (8) et (13), assurément dans (9), tout à fait dans (11), en harmonie avec dans (12), aimer bien dans (14), (volontiers + vouloir bien) dans (15), effectivement dans (16 $\alpha-2)$ et $(16 \beta-2)$, (pourtant + quand même) dans (16 $\beta-3)$. Témoin de l'homogénéité du prédicat qui se substitue à bien, il a toujours comme arguments internes ${ }^{23}$ faire DC sentiment positif.

$\overline{22}$ On nous a fait remarquer avec raison que cette valeur de degré élevé ou de haut degré que nous paraphrasons par très vient de l'application de bien à un adjectif gradable. On suggère ainsi que c'est la valeur prototypique ou centrale de bien qui produit le haut degré par combinaison avec la propriété gradable de l'adjectif : bien / adj gradable $\rightarrow$ adj à un degré satisfaisant $\rightarrow$ adj à un degré élevé. On peut en effet, pensons-nous, le vérifier en appliquant bien à un adjectif non gradable : on ne peut pas dire *Je vous trouve bien honnête. L'honnêteté n'est pas gradable. On est honnête ou on ne l'est pas. Et si on peut dire Il est très honnête c'est que très dans ce cas ne renvoie pas à l'intensité ou au degré mais à la réalité de la qualité, correspondant à Il est vraiment honnête.

23 Au sens de Oswald Ducrot et Marion Carel. Tout prédicat correspond à un bloc sémantique décomposable en deux arguments internes reliés par donc (DC) ou pourtant (PT). Ces arguments internes, lexicalement différents du 
On peut donc considérer que tous les emplois de bien ont un premier niveau commun de complexité, une sorte de seuil minimal dans la construction du sens qui associe l'occurrence de bien à l'occurrence d'un faire qui génère une réaction positive. Léquivalence sémantique se manifeste à travers une relation formelle de substitution, à une position syntaxiquement stable de la chaîne parlée. En partant de ce seuil, qui présente pour ainsi dire un degré zéro de complexité, les emplois se complexifient en fonction du nombre et de la nature des réductions appropriées nécessitées par l'actualisation du prédicat.

Une équivalence peut, comme on vient de le voir, s'établir entre une valeur que prend un constituant $x$ - en l'occurrence bien - dans un contexte syntaxique et lexical donné et une structure syntaxique et des constituants indépendants de ce constituant $x$. Dans ce cas, l'équivalence sert à définir précisément et à délimiter les propriétés de l'une des valeurs de $x$ parmi un éventail de valeurs possibles. Elle donne ainsi une assise lexicale et syntaxique à chacune des réalisations sémantiques de la polysémie de $x$. Mais elle le fait avec des constituants nouveaux et même, souvent, avec des structures nouvelles, c'est-à-dire avec des constituants et des structures qui ne peuvent pas être dérivés régulièrement du constituant $x$ et de ses constructions définitoires usuelles. C'est la raison pour laquelle on peut considérer que toutes les fois où on établit, dans le cadre d'une distinction des valeurs polysémiques, une équivalence entre la suite de mots qui contient $x$-, par exemple c'est bien - et la suite qui exprime l'une des valeurs $x$ ' de l'éventail des valeurs polysémiques de $x$-par exemple, c'est une bonne chose, on exprime les conditions formelles d'un degré d'éloignement de la valeur $x$ ' sélectionnée dans un éventail de valeurs possibles de $x$. Ainsi ce degré est zéro dans le cas de la relation $(6 a) \leftrightarrow\left(6 a^{\prime}\right)$, mais il est maximal dans le cas de la relation $(16) \leftrightarrow(16 ’ \beta-3)$. Ce degré d'éloignement est mesurable parce qu'il correspond à des variations formelles dont on

prédicat, constituent en quelque sorte des primitives sémantiques qui expliquent en grande partie la relation du prédicat à ses actualisateurs et aux autres prédicats dans la chaîne du discours. (Voir Amr Helmy Ibrahim, 2001, op. cit., p. 92-126.) 
peut évaluer la régularité. Une relation relativement régulière en français comme celle qui régit le passage d'un adverbe à un adjectif comme dans bien à bon(ne) $\left[(1 / 2 / 3 / 7) \leftrightarrow\left(1 a^{\prime} / 2 a^{\prime}\right.\right.$ / 3a' /7a') ], ou qui n'est que le résultat de l'application d'un procédé comme l'antiphrase - par exemple dans (13a) $\leftrightarrow(13 \mathrm{a})$ - présentera un degré d'éloignement moindre que celle qui régit le passage de (4a) à (4a'). On peut également en évaluer le coût en termes de production de formes nouvelles ou de variations dans les formes existantes. Ainsi donc, en partant de la connaissance de l'entrée lexicale - en l'occurrence bien - et de ses constructions usuelles - voir les chiffres prime -, il est tout à fait possible de déterminer précisément le nombre et la nature des opérations nécessaires pour obtenir la suite équivalente qui correspond à l'une des valeurs d'un éventail gradué de polysémie. Or, l'observation synoptique de cet éventail révèle, dans ce cas, une corrélation étroite entre la complexité - nombre et nature des opérations nécessaires pour parvenir à une équivalence - et un certain degré d'éloignement entre les deux termes de l'équivalence, la distance qui autorise à parler d'éloignement étant appréciée à l'aune de la continuité morphologique et structurelle - tout changement morphologique ou structurel ayant pour effet d'accroître automatiquement la distance.

On peut donc en conclure que l'extension de la polysémie d'une expression consiste, paradoxalement, à l'éloigner de son foyer tout en maintenant une relation d'équivalence avec ce foyer, autrement dit que cette extension a une tendance foncièrement centrifuge. Ce qui est remarquable n'est pas, bien entendu, le caractère centrifuge de la polysémie, mais le fait que ce caractère soit exprimable et mesurable dans le cadre d'une équivalence. Que le mode opératoire de la fonction différenciatrice de la polysémie soit inclus dans des mécanismes qui garantissent l'équivalence, c'est-à-dire l'égalité de deux sommes de constituants dont la nature, le nombre et les relations qu'ils entretiennent sont différents. 


\subsubsection{Relations d'appropriation et extension centripète des classes d'équivalence}

L'élasticité de la langue peut néanmoins jouer autrement. La tendance d'un énoncé $x$ 'à se différencier d'un énoncé $x$ auquel il est équivalent par sa structure et son sens, peut être centripète. $x$ 'ne se distingue pas alors de $x$ par une distance qu'il institue par rapport à la valeur sémantique et à la construction usuelle de $x$ mais, au contraire, par une délimitation de $x$ qui en donne une définition analytique correspondant à un usage suffisamment contextualisé pour qu'il ne puisse pas être ambigu. La définition analytique restrictive qui rend dans ce cas $x$ et $x$ ' équivalents part d'un mouvement opposé à celui, centrifuge, que produit la polysémie, puisqu'il rend $x$ monosémique, d'où sa qualification de centripète.

Les relations d'appropriation commandent deux types distincts d'extension centripète : celui que l'on observe dans les dérivations analytiques d'origine méronymique qu'on retrouve principalement dans les constructions à support et celui qui résulte de l'asymétrie constitutive des distributions complémentaires.

\subsubsection{Extension centripète par dérivation méronymique : les constructions à supports}

Lorsque l'un des termes d'une équivalence linguistique définit de manière analytique l'autre, il contient toujours une relation asymétrique d'appropriation entre au moins deux de ses constituants comme on peut le voir dans le fonctionnement grammatical et sémantique de la relation entre le nom prédicatif et le verbe support. Observons les énoncés suivants.

Idrisse m'a garanti (son soutien + ma voiture).

(1a) Idrisse m'a (donné + fourni + ${ }^{*}$ fichu) $(($ une + la $)$ garantie de son soutien $+\left(\right.$ des $+{ }^{*}$ les $)$ garanties (concernant son soutien $\left.\left.+E\right)\right)$. (1b) Idrisse m'a (donné + fourni + $\left.{ }^{*} f i c h u\right)\left(\right.$ une $+{ }^{\#} l a^{24}+$ des +

$\overline{24}$ “\# « indique que le mot qui le suit n'est acceptable ou grammatical dans l'énoncé où il se trouve qu'avec un sens différent de celui qui fait l'objet de l'analyse. En l'occurrence, avec de les verbes donner ou fournir ne sont plus des verbes supports, l'énoncé (5a') Idrisse m’a (donné + fourni) (une + la) garantie de la voiture signifiant m'a (remis + passé) un document de garantie de 
"les) garantie(s) (pour $+{ }^{*}$ de) la voiture.

Sonia m'a conseillé.

(2a) Sonia m'a (donné + fourni + *fichu) (un conseil + des conseils $+{ }^{*}$ le conseil $+{ }^{*}$ les conseil $\left.{ }^{25}\right)$.

Charles mia téléphoné hier.

(3a) Charles m'a (donné $+{ }^{*}$ fourni $+{ }^{*}$ fichu) $([u n+$ des $]$ coup $(s)$ de téléphone + + *le coup de téléphone + *les coups de téléphone) hier.

Léon m’a (expliqué la situation + tout expliqué).

(4a) Léon m'a (donné + fourni + *fichu) (une explication de + l'explication de + des explications concernant + les explications concernant) la situation.

(4b) Léon m'a (donné + fourni + *fichu) (une explication sur tout ce qu'il se passait + l'explication de tout ce qu'il se passait + des explications concernant tout ce qu'il se passait + les explications concernant tout ce qu'il se passait).

la voiture. Transmettre un document de garantie d'un objet n'est pas équivalent au fait de garantir cet objet. [Par souci de clarification terminologique et afin d'éviter toute confusion, lorsqu'un verbe a, selon les contextes, un emploi de verbe support et un emploi qu'on aurait tendance à qualifier intuitivement de "normal " ou " habituel » et eu égard au caractère très trompeur et extrêmement polysémique des termes normal ou habituel dans ce contexte, nous appelons les emplois du type (5a') distributionnels par opposition aux emplois support, opérateur, figé, etc.

25 Le conseil et Les conseils sont, bien entendu, grammaticaux et acceptables, de même que d'autres occurrences dans certains de nos exemples de ces déterminants "définis " lorsqu'ils ont une fonction anaphorique claire et renvoient à une occurrence antérieure toujours présente dans la situation d'interlocution du nom qu'ils déterminent. Il s'agit, dans ce cas, d'une fonction à la fois anaphorique et déictique correspondant à " le conseil dont il a été fait mention » ou "dont il a été question ». Cette fonction anaphorique ou déictique attachée à ce type de déterminant en français, peut elle aussi être bloquée par la nature du nom qui soit change de sens - par exemple en (1a) assurance s'il n'est pas suivi de son soutien - soit ne peut pas du tout être repris - par exemple, en (7a), (7b) et (8a) baiser ou jouissance. Mais elle n'en reste pas moins très générale et comme elle est exclusivement situationnelle ou contextuelle, c'est-à-dire qu'elle peut s'ajouter à n'importe quel énoncé avec le même résultat. Nous ne la prenons pas en compte ici afin de mieux départager ce qui relève des contraintes lexicales et structurelles de ce qui ne relève que de contraintes situationnelles ou discursives extérieures à la phrase simple. 
Idir a giflé Zoé.

(5a) Idir a (donné + *fourni + fichu) (une + *la + ?des + *les) gifle(s) à Zoé.

Chloé m'a embrassé sur les lèvres.

(6a) Chloé m’a (donné + *fourni + *fichu) $\left(u n+{ }^{*}\right.$ des + *le + *les) baiser(s) sur les lèvres.

(6b) Chloé a (posé + plaqué) (un + ${ }^{*}$ des $+{ }^{*} l e+{ }^{*}$ les $)$ baiser(s) sur mes lèvres.

Chloé m'a fait jouir comme jamais.

(7a) Chloé m’a (donné $+{ }^{*}$ fourni $+{ }^{*}$ fichu) $\left(\right.$ une $+{ }^{*}$ des $+* l a+$ *les) jouissance(s) sans précédent.

(7b) Chloé m'a procuré une jouissance sans précédent.

Le comportement de Marouane m'a suggéré de vous appeler.

(8a) Le comportement de Marouane m'a (donné + *fourni + * fichu) ('une $+l '+{ }^{*}$ des $+{ }^{*}$ les) idée de vous appeler.

La persévérance d'El Chérif (impressionne + mimpressionne).

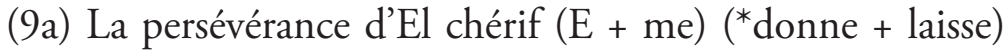

(une $+{ }^{*} \mathrm{la}+{ }^{*}$ des $+{ }^{*}$ les) forte(s) impression(s).

C'est au roi seul de donner le signal du départ, le coup d'envoi, de cette manifestation.

(10) Le signal du départ, le coup d'envoi, dans cette manifestation, est de la compétence exclusive du roi.

(10a) Il n'y a que le roi qui ait la compétence de (donner + *fournir + *ficher) [(un + le + ?des + ?les) signallaux de départ $]$ $+\left[\left(u n+l e+{ }^{*}\right.\right.$ des $+{ }^{*}$ les $) \operatorname{coup}(s)$ d'envoi $]$ (de cette + dans ce type de) manifestation.

Son coup de pied mia abîmé le tibia.

(11) $($ Son + Ses) coup $(s)$ de pied m'(a+ont) abîmé le tibia. (11a) ("un26 + le + "des + les) coup(s) de pied qu'il m'a (donné(s) + *fourni(s) + fichu(s)) m'alont abîmé le tibia.

Nous constatons, en observant ces exemples, cinq types de problèmes indépendants qui ont pour dénominateur commun la nature lexicale du nom prédicatif et son rapport à son actua-

26 Avec un l'équivalence est avec Il y a eu un coup de pied (de sa part + venant de lui) qui mia abîmé le tibia. 
lisateur principal, le verbe support. Chacun de ces problèmes constitue un type ou un sous-type différent de complexité due à une relation d'appropriation.

\section{/I/ La possibilité ou non de substituer au verbe donner, un autre verbe support avec des valeurs plus spécifiques : fournir,} ficher, voire poser, plaquer (6b), laisser (9a), ou même des verbes qui, tout en continuant à fonctionner comme des verbes supports, analysent une expression causale habituellement grammaticalisée par un opérateur : procurer $(7 \mathrm{~b})$.

\section{/II/ Le choix du déterminant approprié au nom prédicatif} à la suite d'un verbe support. La distribution des déterminants indéfini singulier, défini singulier, pluriel indéfini, pluriel défini, partitif, possessif singulier, possessif pluriel, déterminant zéro ou absence de déterminant connaît globalement, lorsque le déterminant est la seule détermination qui s'applique au nom prédica$\mathrm{tif}^{27}$, - à l'exception notable des noms prédicatifs de sentiment ou causatifs de sentiment comme amour ou tristesse qui ont un partitif comme déterminant de base et d'une petite liste fermée de prédicats de sensations physiques élémentaires qui ne prennent pas de déterminant ${ }^{28}$ - une tendance dominante : l'usage d'un déterminant indéfini, accessoirement du possessif, et, le plus souvent au singulier: (Ils ont déclaré la guerre à la Russie $\leftrightarrow$ Ils ont fait une déclaration de guerre à la Russie / Il n'en a pas parlé (quand il a fait + dans) (son + sa) (intervention + exposé + communication + conférence) / Ils se sont réunis $\leftrightarrow$ Ils ont tenu une réunion / Il finira bien par se décider $\leftrightarrow$ Il finira bien par prendre une décision I Il m'a conseillé $\leftrightarrow$ Il m'a donné (un + des) conseil(s) / Léon va inviter toute la ville $\leftrightarrow$ Léon va lancer (une + des) invitation(s) à toute la ville /. Cette forme de détermination apparaît également dans les cas où la construction à support n'est pas liée à une nominalisation : Il lui a fait un cadeau / Il lui a mis une mauvaise

$27 \quad$ Par exemple, Il éprouve de la haine où le nom prédicatif haine n'est déterminé que par le déterminant partitif de la, opposé à Il éprouve une haine tenace où haine est déterminé à la fois par le déterminant indéfini une et par l'adjectif tenace.

J'ai (chaud + faim + froid + mal + soif). 
note / Il éprouve un malaise / Il donne une fête / Il passe un(e) (concours + examen + test + épreuve) / Il a commis un(e) (crime + délit + faute + erreur), etc. La tendance est inverse dans les constructions figées où on a, soit un déterminant défini, le plus souvent également au singulier, soit, et c'est le cas le plus fréquent, une absence de déterminant. Par exemple, pour dire qu'on a construit un mur à la limite d'une propriété on dira qu'on a monté ou fait un mur à cet endroit; la substitution de le à un produira automatiquement - si on n'est pas dans une situation de reprise anaphorique - un important changement de sens avec émergence d'une construction figée $\grave{A}$ la caserne, je faisais le mur tous les soirs pour la voir; au mieux, une interprétation ambiguë Quand on m'a surpris, je faisais le mur. Parallèlement, l'équivalence entre Maintenant, il va falloir murer la fenêtre et Maintenant il va falloir (boucher + fermer) la fenêtre par un mur devient impossible si l'on substitue le à un. Mais on n'est pas face à une règle absolue comme le montrent nos exemples (1a / 1b), (4a / 4b), (8a), (9), (9a), et (10a). Les cas qui démentent la tendance générale s'expliquent par la structure sémantique interne du nom prédicatif. Cette structure sémantique interne est, au même titre que les contraintes grammaticales, analysable dans les termes d'un lexique définitoire élémentair ${ }^{29}$, et présente le même degré de complexité que présente la combinatoire de ce lexique élémentaire.

\section{/III/ Le type de construction syntaxique qui autorise une équivalence entre la construction à support et la construction} sans support. Par exemple, le fait que tous les arguments potentiels d'un prédicat verbal ou nominal soient ou non exprimés peut avoir, comme dans les séries [1], [8] et [9] une incidence directe sur les conditions de restructuration et d'équivalence des énoncés. L'importance de ce phénomène est accentuée par son lien avec les mécanismes de reconstruction des énoncés réduits. Nos exemples [1] en donnent une bonne représentation. Ainsi, un verbe comme garantir peut avoir au moins deux sujets

\footnotetext{
29 Voir Amr Helmy Ibrahim, "L'analyse matricielle définitoire... ", à paraître, op. cit.
} 
internes : un quelqu'un "quelconque " et un quelqu'un "qui est un garant "; il peut aussi avoir deux objets internes en construction directe : quelque chose et quelqu'un. Or, l'une des propriétés cruciales des constructions à supports est qu'elles sélectionnent nécessairement une et une seule des structures potentielles de la construction verbale, ce qui revient à dire qu'elles construisent leur sens à partir des sujets et objets internes de cette construction. Ces constructions ont donc moins de chances d'être ambiguës et généralement elles ne le sont pas. Une ambiguïté moindre ou une absence d'ambiguïté peut avoir, au regard de la complexité, un effet paradoxal. En effet, la production d'une ambiguïté dont on est conscient et dont on joue implique des mécanismes de maîtrise de la gestion du sens plus complexes que ceux qui sont impliqués par la production d'un énoncé non ambigu. Mais, parallèlement, la production consciente d'un énoncé analytique non ambigu implique, dès lors qu'il est sélectionné parmi un grand nombre d'autres énoncés analytiques possibles, un nombre plus grand de choix et donc une gestion plus sophistiquée de la complexité que la production d'un énoncé synthétique plus court et en apparence plus simple dont on ne mesure pas l'ambiguïté. La mesure de la complexité ne prend dans ce cas son sens que si elle est ramenée au degré de conscience que le locuteur a de sa gestion du sens qu'il construit.

\section{/IVI L'existence de situations où donner se trouve à la fron- tière d'un verbe support et d'un verbe causatif. Comme nous} l'avons décrit plus systématiquement ${ }^{30}$, une même entrée verbale, en l'occurrence donner, peut avoir une valeur distributionnelle : Il m'a donné (ses clés + 300 euros + un cadeau + ...), une valeur d'opérateur causatif : Ce que tu dis me donne (la frousse + à réfléchir...+$)$ que l'on peut toujours paraphraser par des constructions du type : Ce que tu me dis fait que (j'ai la frousse + je réfléchis...+$)$, une valeur de verbe figé : Il va nous donner pas mal

$\overline{30}$ Amr Helmy Ibrahim, "Une classification des verbes en 6 classes asymétriques hiérarchisées ", Syntaxe et Sémantique 2, dans Françoise Cordier, Jacques François et Bernard Victorri (dir.), Sémantique du lexique verbal, Caen, Presses universitaires de Caen, 2000, p. 81-98. 
de fil à retordre, et une valeur de verbe support comme dans nos exemples. Mais certains de ces exemples (7a, 8a, 9a) peuvent amener à se demander si donner est un verbe support ou un opérateur causatif, notamment lorsqu'une paraphrase en faire est possible comme c'est le cas en (7a) $A$ fait jouir $B \leftrightarrow A$ donne de la jouissance à $B$, ou en (8a) $A$ (fait germer) une idée dans la tête de $B$ ou $A$ fait que $B$ a une idée $\leftrightarrow A$ donne une idée à $B$, ou dans (9a) : A fait que $B$ a l'impression que $x \leftrightarrow A$ donne à $B$ l'impression de $x$. On retrouve ici une problématique non résolue dont les termes ont été très clairement posés, à notre connaissance pour la première fois, à partir du français mais dans une perspective et avec une méthodologie qui vaut pour toute la famille indoeuropéenne, par les travaux de Maurice Gross dès les années 1975-1978 et de Frédérique (Yannick) Gheerbrant ${ }^{31}$ sur les verbes et les noms dits de "sentiment " ou "psychologiques". L'argumentation, développée, vérifiée et critiquée par une application systématique à un ensemble important d'entrées lexicales par Gheerbrant montre que cet ensemble croise plusieurs niveaux d'ambiguïté qu'il est souvent difficile, pour ne pas dire impossible, de dissocier. Il est probable que la difficulté soit due au fait que dans les langues indo-européennes l'évolution des formes grammaticales a souvent rompu presque définitivement, comme l'a souligné le premier Wilhelm von Humboldt ${ }^{32}$, le lien qui attachait ces formes aux notions qui les avaient motivées. Paradoxalement, on peut en effet se faire plus facilement une idée de la difficulté en l'illustrant par sa manifestation dans une langue appartenant à une famille réputée plus conservatrice en termes d'évolution comme l'arabe. L'opérateur causatif s'y manifeste sous deux formes bien distinctes.

\footnotetext{
31 Maurice Gross, Méthodes en syntaxe : régime des constructions complétives, Paris, Hermann, 1975, 414 p.; Frédérique (Yannick) Gheerbrant, La nominalisation et les verbes de sentiment, Thèse de doctorat de $3^{\mathrm{e}}$ cycle, Université Paris 7 , 1978, 323 p.

32 Wilhelm von Humboldt, "La recherche linguistique comparative dans son rapport aux différentes phases du développement du langage ", Introduction à l'ouvre sur le kavi et autres essais, [traduction française par Pierre Caussat d'un mémoire lu par Humboldt à l'Académie de Berlin le 29 juin 1820], Paris, Seuil, [1820], 1974, p. 82-84.
} 
D'une part, une construction faisant intervenir l'équivalent de l'opérateur causatif faire, par exemple :

$$
\text { جعلت الخيط يَخُل (فحى) سُمّ الإبرة }
$$

Jacalat 'al khayt:a yadkholo ( $\mathrm{f} i$ ) sommi -l ibra A fait le fil entrer (dans) chas l'aiguille (elle) a fait (que le fil entre dans le chas de l'aiguille + entrer le fil dans le chas de l'aiguille)

D'autre part, un recours à un schème verbal - أفُْعَ 'af'al - qui a vocation à exprimer la cause :

أدخلت الخيط (فهى) سُمّم الإبرة

'adkhalat 'al khayt:a (fî) sommi -l ibra a entré le fil (dans) chas l'aiguille

(elle) a entré le fil (dans) le chas de l'aiguille

L'arabe donne une morphologie spécifique aux verbes qui prennent un sens causatif ${ }^{33}$, alors qu'en français entrer garde la même morphologie qu'il soit non causatif comme dans Amr est entré ou causatif comme dans Amr a entré le fil dans le chas de l'aiguille. Mais, dans ce cas particulier, le français, comme l'arabe, distinguent syntaxiquement les deux valeurs d'entrer en opposant une construction intransitive - sémantiquement non causative - Quelqu'un entre - à une construction transitive - sémantiquement causative - Quelqu'un entre quelque chose. On peut donc, sous réserve d'inventaire - celui de tous les verbes qui fonctionnent en français comme entrer et celui de tous les verbes arabes à sens causatif - faire une première constatation : il existe en français des verbes sémantiquement causatifs qui ne sont pas marqués morphologiquement, alors qu'ils le sont dans d'autres langues, et qu'on ne peut identifier formellement que par une procédure syntaxique qui n'a de sens que s'ils ont un homonyme non causatif, étant donné qu'il est impossible d'associer la transitivité à la causation et l'intransitivité à la non causation. Autrement dit, on peut déjà affirmer à ce stade qu'en français,

33 En arabe, le verbe دَخَل dakhala (= entrer) dans دَخَلَ عمرو dakhala Amr (=Amr est entré) prend le schème فَ فَلَ facala et ne peut jamais avoir un sens causatif. 
les causatifs de type entrer dans (13a) sont des causatifs internes, c'est-à-dire qu'ils ne sont analysables qu'à travers les restructurations qui les relient à leur matrice. Si maintenant on revient à (7) en substituant au verbe jouir le verbe (se) réjouir qui en est étymologiquement issu et qui lui est toujours, même si c'est sur un registre plus atténué, sémantiquement apparenté, on peut construire la série suivante :

(14) (Ta réussite réjouit Ali + Ali se réjouit de ta réussite)

(14a) Ta réussite fait que Ali se réjouit.

(14b) Ta réussite (donne + fournit) à Ali (une + une bonne + des) raison(s) de se réjouir.

(14b') Ta réussite (donne + fournit) (une + une bonne + des) raison(s) à la réjouissance de Ali.

(14c) Ta réussite (donne + fournit) à Ali une cause de réjouissance $\leftrightarrow$ Ta réussite (donne + fournit) une cause à la réjouissance de Ali.

(14c') Ta réussite (*donne + ${ }^{*}$ fournit $+{ }^{*}$ fait + est) la cause de la réjouissance de Ali $\leftrightarrow$ Ta réussite cause la réjouissance de Ali.

(14d) Ta réussite fait l'objet de la réjouissance de Ali $\leftrightarrow L a$ réjouissance de Ali a pour objet ta réussite.

D'où il ressort que la nominalisation du verbe (se) réjouir : (14b'), (14c), (14c') et (14d), qui est, ainsi qu'en témoigne (14a), un causatif interne, fait apparaittre à travers la famille des restructurations qui décrivent le mode d'actualisation du nom prédicatif réjouissance, une équivalence entre la construction causative de référence (14a) faire que $N \mathrm{seV}$ et la construction à verbe support et nom opérateur générique ${ }^{34}$ qui décrit le nom prédicatif: $V_{-s u p}$ Det $N_{- \text {opér géner }}$ de $\left(N_{- \text {pred }}+V\right)$ cf. (14b), (14b'), (14c) et (14c'). Si (14a) explique (14) tout en lui étant équivalent c'est

34 Les verbes supports $V_{\text {-sup }}$ sont dans ces exemples : donner, fournir et être; les noms opérateurs génériques : raison et cause. Les noms opérateurs génériques, réductibles, au même titre que les supports, diffèrent des supports en ce qu'ils ne sont pas appropriés aux prédicats auxquels ils s'appliquent qui eux ne sont pas réductibles. La série 14 pourrait être réduite à «La réjonissance de Ali? - Ta réussite! ». 
parce que (14) doit être décrit dans les termes d'un groupe actualisateur du prédicat réjouissance formé du verbe support donner ou de son extension fournir et du nom opérateur générique raison ou cause.

Si nous observons l'énoncé (14d), synonyme des précédents, nous remarquerons qu'au groupe actualisateur du type donner une raison à ou être la cause de s'est substitué le groupe actualisateur a pour objet ou sa forme converse fait l'objet de. C'est donc dire qu'au groupe actualisateur caractéristique de la causalité interne s'est substitué le groupe actualisateur caractéristique de la transitivité. On comprend mieux ainsi l'ancrage de la causalité interne dans la transitivité et on s'explique mieux la difficulté qu'il peut y avoir dans certains contextes frontaliers à départager les interprétations portées par un opérateur causatif sous-jacent de celles qui sont portées par un simple support d'actualisation. Certains prédicats nominaux sélectionnent, en effet, des groupes actualisateurs composés d'un verbe support et d'un nom opérateur générique de type causatif et deviennent équivalents à un " groupe support de finalité » - faire l'objet de / avoir pour objet - où la relation transitive apparente ne distingue pas entre le verbe opérateur causatif faire suivie du nom opérateur objet et le verbe support avoir suivi du même nom opérateur objet.

Cette description analytique montre, entre autres, dans quelles conditions se construit un sens causatif et comment l'expression de la cause s'inscrit dans un continuum qui n'est pas commandé par le notionnel - l'idée de cause -, mais par la généalogie de l'usage des outils linguistiques nécessaires à l'actualisation d'un prédicat.

Il n'est pas indifférent, comme on le constate en (14b), que le français mobilise alternativement bonne ou le pluriel pour déterminer le nom opérateur générique raison, qu'il établisse une distribution complémentaire entre donner et laisser (9a), qu'il fasse de fournir un support redondant de donner dans un nombre limité de configurations, enfin qu'il établisse une relation converse ${ }^{35}$ entre donner et avoir. La complexité de la relation qui

$\overline{35} \mathrm{Au}$ sens défini par Gaston Gross (Les constructions converses du français, 
lie le nom prédicatif au support qui l'actualise tient dans le fait que des constituants potentiellement nouveaux sont créés à travers des séries de reconfigurations du même, c'est-à-dire des redondances. Et c'est le degré de redondance qui est mesurable.

/VI L'existence d'équivalences où la translation se manifeste formellement par une dérivation morphologique régulière parallèlement à des équivalences où il n'y a pas de dérivation morphologique liée à la translation comme c'est le cas entre (6), embrasser, et (6a), donner un baiser, ou (6b), (poser + plaquer) un baiser, (9) suggérer et (9a) donner l'idée.

Ici, la représentation de la continuité sémantique entre (7) Chloé m'a embrassé et (7a) Chloé m'a donné un baiser sur les lèvres ou (7b) Chloé a (posé + plaqué) un baiser sur mes lèvres ou entre (9) Le comportement de Marouane m'a suggéré de vous appeler et (9a) Le comportement de Marouane m'a donné l'idée de vous appeler, sans contrepartie morphologique, repose entièrement sur la perception intuitive que l'équivalence syntaxique de deux structures $\left(\left[N_{\text {-hum }}+N\right.\right.$ de $\left.N_{\text {-hum }}\right] V_{\text {(embrasser + suggérer) }}\left[N_{\text {i-hum }}+\right.$ à $N_{\text {i-hum }}$ de $\Omega] \leftrightarrow N_{\text {-hum }} V_{\text {-sup donner }} \operatorname{Det}\left[N_{\text {-pred }}+N_{\text {-op }}^{\text {-hum }}\right]$ [sur $\operatorname{Det} N_{\text {-pc }}\left(\operatorname{de} N_{\text {i }}\right)$ + de $\Omega$ ], vérifiée dans d'autres cas, est généralisable au cas observé et entraîne de ce fait une équivalence sémantique bien qu'il n'y ait sémantiquement aucun rapport entre donner un baiser et donner une idée.

5.3.2.2. Extension centripète des formes équivalentes asymétriques: les distributions complémentaires

Cette dernière configuration - /V/ - n'est pas rare. Il existe dans toutes les langues des dérivations formelles qui expriment une continuité sémantique sans qu'il soit possible de coupler selon une procédure généralisable un mécanisme syntaxique et une dérivation morphologique. C'est le cas, par exemple, du phénomène bien connu de distribution complémentaire dont la paire très / beaucoup constitue en français un modèle :

Genève-Paris, Droz, 1989, 513 p.). 
[a] J'ai beaucoup (travaillé + souffert) sans rien dire. // J'ai beaucoup couru.

[b] J'ai été très (malade + fatigué) sans rien dire. // J'ai couru très vite.

Les deux mots ou lexèmes beaucoup et très ne sont pas substituables l'un à l'autre pour des raisons strictement grammaticales ou syntaxiques :

[a'] *J'ai très (travaillé + souffert) sans rien dire. // * J'ai très couru.

[b'] *J'ai été beaucoup (malade + fatigué) sans rien dire // *J'ai couru beaucoup vite.

Les termes interdits en [a'] et [b'] sont intéressants du fait qu'ils sont synonymes de ceux qui sont autorisés en [a] et [b] et que tous appartiennent à la même catégorie grammaticale ou partie du discours ainsi qu'en atteste leur équivalence de distribution avec d'autres constituants de cette catégorie, par exemple ici trop:

[c] J'ai (beaucoup + longtemps + trop) (travaillé + souffert) sans rien dire. // J'ai [(beaucoup + trop) couru] + [couru longtemps]

[c'] J'ai été (très + longtemps + trop) (malade + fatigué) sans rien dire. // J'ai couru [(très + trop) vite] + [vite longtemps].

Une équivalence sémantique et catégorielle qui ne s'accompagne pas d'une identité de distribution - très et beaucoup - et une équivalence catégorielle qui s'accompagne d'une identité de distribution mais sans équivalence sémantique - trop et beaucoup / très - peut s'exprimer lexicalement sous la forme suivante :

[a-m] J'ai fourni une grande quantité de travail $\leftrightarrow$ J'ai fourni une quantité importante de travail.

(J'ai couru longtemps + jai passé un temps important à courir)

[b-m] Ma maladie est à un état avancé $\leftrightarrow$ Ma maladie est à un état grave $\leftrightarrow$ L'état (d'avancement + de gravité) de ma maladie a été jugé important.

J’ai couru à une vitesse jugée (importante + élevée) 
[ab-m"] (La quantité de travail que j'ai fournie + Le temps que j'ai passé à courir + Mon état de maladie + Le jugement porté sur la vitesse à laquelle j'ai couru) présente un baut degré d'importance.

On voit à travers ces équivalences le dénominateur commun qui justifie que ces expressions restent sémantiquement équivalentes alors qu'elles n’ont pas la même distribution : le haut degré d'importance, qu'il soit donné, constaté ou jugé.

Mais on voit aussi l'origine des incompatibilités: *une quantité (avancée + grave $) / *$ (un état + une vitesse + un temps) (en grande quantité + en quantité importante), à savoir les contraintes de sélection, ainsi que les conditions d'appropriation qui les accompagnent, de quantité, état, vitesse et temps, qui sont des constituants terminaux des constructions matricielles de $\left(V_{\text {-êre }}\right.$ très $A d j+V$ très $A d v)$ ou $V$ beaucoup.

En comparant les chemins par lesquels sont résolues les différentes incompatibilités de voisinage distributionnel, on retrouve les formulations qui contiennent le dénominateur commun - le haut degré d'importance - et on a une mesure unifiée de la complexité de la construction grammaticale de ces chemins qui sont des matrices analytiques définitoires où le choix des constituants terminaux est étalonné.

On est donc fondé à établir une gradation objective, dans la mesure de la complexité, en fonction des choix qui reconstituent des relations adéquates d'appropriation lexicale. En effet, les mécanismes d'appropriation que nous avons décrits dans les constructions qui présentent des équivalences asymétriques, qu'il s'agisse de constructions à supports ou de constructions à distributions complémentaires sont parmi les plus complexes. Mais ces relations, dès lors qu'elles sont décrites en termes de reconstitution et de réduction de constituants définitoires et d'actualisateurs comme les verbes supports, les noms classifieurs, les corrélats, les noms génériques, les verbes et noms opérateurs, c'est-à-dire d'outils descriptifs actualisateurs redondants, deviennent mesurables. De plus, ces outils ont le mérite d'être universels. Ils transcendent les catégories grammaticales 
particulières d'une langue donnée et sont transposables dans n'importe quelle langue du monde.

Du fait de leur discontinuité morphologique et grammaticale, les distributions complémentaires soulèvent des problèmes particulièrement complexes. Or, elles peuvent toucher des prédications d'un usage très courant, par exemple dormir et manger. L'analyse matricielle révèle alors vite le caractère faussement simple de ces notions et de leur expression linguistique. Elle permet également d'expliquer les raisons qui amènent parfois l'usage ordinaire à rompre dans certaines langues la continuité morphologique et grammaticale de leur champ lexical.

\subsection{Nominalisation de manger}

Il n'existe pas en français de nom d'événement ou de nom d'action morphologiquement dérivé du verbe manger et qui lui soit sémantiquement équivalent. Il y a, bien sûr, manducation, mais ce terme, bien que régulièrement dérivé de la base latine de manger, est aujourd'hui un terme spécialisé de la physiologie et ne recouvre plus qu'une partie du sens de manger. Il n'est donc pas facile dans ces conditions, si l'on veut respecter l'esprit de l'analyse matricielle où la forme terminale du prédicat dans la matrice est nécessairement nominale, de faire le tri entre les énoncés asymétriques complémentaires correspondant à Je mange une cuisse de poulet dont voici l'essentiel :

[a-1] Je mets à plusieurs reprises dans ma bouche des morceaux d'une cuisse de poulet.

[a-2] Une cuisse de poulet est un(e) (aliment + nourriture + substance mangeable).

[a-3] J'ingère cet(te) (aliment + nourriture + substance mangeable) par manducation.

[b-1] Je m(e) (alimente + nourris) avec un(e) (aliment + nourriture + substance mangeable).

[b-2] Cet(te) (aliment + nourriture + substance mangeable) est une cuisse de poulet. 
[b-3] (J'introduis + Je mets) à plusieurs reprises dans ma bouche des morceaux de cette cuisse de poulet qui est un(e) (aliment + nourriture + substance mangeable).

[b-4] (J'exerce + J'accomplis) dans ma bouche une action de manducation de ces morceaux de cuisse de poulet qu'ensuite (j'ingère $+\mathrm{j}$ 'avale).

[c-1] Je m(e) (alimente + nourris).

[c-2] Pour cela je prends, mastique et ingère par petites quantités des (substances + aliments + nourritures) mangeables.

[d-1] Je prends pour (mon alimentation + nourriture) des quantités mangeables de cuisse de poulet.

[d-2] J'ingère ces quantités par manducation.

[e-1] Je me livre à l'activité de prendre et de mettre dans ma bouche de petites quantités d'une cuisse de poulet qui est un(e) (aliment + nourriture + substance mangeable). [e-2] J'ingère ce qui a fait l'objet d'une manducation.

Le tri parmi ces énoncés peut se faire en suivant le protocole de l'analyse matricielle :

/1/ retrouver à travers les énoncés asymétriques complémentaires la totalité du sens de manger en dégageant ce qui en est actualisé dans l'énoncé observé;

/2/ partir d'un foyer prédicatif;

/3/ aboutir à une relation de dérivation morphosyntaxique simple entre la matrice et l'énoncé observé, ce qui suppose de pouvoir justifier la réduction de ce qui apparaît dans la matrice sans apparaître dans l'énoncé observé.

L'application du protocole d'analyse montre alors la très grande complexité de ce verbe apparemment très simple, acquis très tôt et d'un usage très courant. Il montre, en effet, que le fait de manger implique nécessairement la réalisation de cinq opérations : 
(a) Le fait de s'alimenter ou de se nourrir. D'où le fait que lorsqu'on dit Il mange mal on peut vouloir dire Il ne mange pas des choses nourrissantes.

(b) Le fait de prendre ce dont on s'alimente ou se nourrit à une ou plusieurs reprises en petite quantité et à le mettre dans sa bouche, donc la manière dont on s'alimente. D'où le fait que lorsqu'on dit Il mange mal on peut vouloir dire Il ne sait pas se tenir à table.

(c) Le fait que ce que l'on met dans sa bouche soit mangeable ou comestible. D'où : Comment peut-on manger une chose pareille?

(d) Le fait de procéder à la manducation - préhension, mastication, insalivation et déglutition - de ce que l'on a dans la bouche, d'où le fait qu'on puisse dire : Il mange bruyamment ou Il fait du bruit en mangeant.

(e) Le fait d'ingérer ce qui a fait l'objet d'une manducation et le statut de celui qui se livre à cette ingestion, d'où $I l$ mange beaucoup ou Il ne mange rien.

Les cinq opérations impliquées sémantiquement par l'usage du verbe n'apparaissent pas de la même manière et au même niveau dans l'actualisation d'une de ses occurrences. Par exemple, une construction intransitive comme Il ne mange plus depuis trois jours non seulement fait remonter au premier plan le fait de salimenter ou de se nourrir mais identifie manger à cette seule opération. Par contraste, dans Ce que j'ai mangé hier m'est resté sur l'estomac, c'est le fait d'avoir ingéré ce qui a fait l'objet d'une manducation qui occupe seul le premier plan, alors que dans $N e$ fais pas de bruit en mangeant, c'est la quatrième opération, la manducation, qui est concernée au premier chef par le fait de manger.

Parallèlement, les sens dérivés, qu'ils soient spécialisés, figurés ou métaphoriques, de manger comme : / $\alpha$ / La rouille mange le fer ou / $\beta /$ Ce chapeau lui mange le visage sont dérivés de l'une des 
cinq opérations à l'exclusion de toutes les autres : / $\alpha /$ dérive de (e) par substitution de faire disparaître à ingérer et de action chimique à manducation $^{36}$, tandis que $/ \beta /$ dérive toujours de (e) mais par substitution de cacher à ingérer et de recouvrement à manducation.

La recherche d'une solution à l'absence d'une base nominale en relation de dérivation régulière avec le verbe correspondant révèle, en fait, la fonction essentielle, dans l'évolution des langues, de ce que l'on pourrait appeler les sélections méronymiques et l'ampleur des déplacements qu'elles opèrent dans la construction du sens. Alors que, en français comme dans toutes les langues que nous connaissons, la base nominale, susceptible d'être prédicative, d'un verbe est le plus souvent d'un sens beaucoup plus général et beaucoup moins défini que le verbe qui lui correspond, le rapport avec manger est inversé. Le nom manducation correspondant au verbe manger n'en est plus, en termes matriciels, la base, mais caractérise une seule des cinq opérations impliquées par le verbe, ce n'est donc qu'une partie du tout. Cette partie, malgré son importance et bien qu'elle continue à donner son nom au tout, peut disparaître, comme on vient de le voir avec (a), (c) et (d), au profit d'une autre partie qui n'a pas laissé de trace formelle dans la forme qui désigne le tout : s'alimenter, se nourrir, faire disparaître, cacher.

\section{Complexité de la concaténation externe du discours}

L'adéquation d'une prédication à une structure énonciative mélodique et signifiante dans les conditions que nous avons décrites établit automatiquement entre deux des constituants de la matrice analytique définitoire de l'énoncé qu'on a produit une relation médiatisée par donc ou pourtant ${ }^{37}$. Ces deux constituants, les arguments internes du prédicat, peuvent être un corrélat, un nom classifieur, un support ou un opérateur. La cohérence des enchaînements des énoncés simples qui se jouxtent ou qui sont voisins est étroitement tributaire d'une articulation sémantique-

\footnotetext{
36 Qui est une action mécanique.

37 Voir note 23.
} 
ment acceptable du produit de la relation des arguments internes de chaque énoncé.

Pour qu'un discours soit perçu comme cohérent, les énoncés simples dont il est formé doivent être perçus comme reliés. Ces liens peuvent être signalés par des constituants dédiés à l'enchaînement, par exemple des connecteurs ou des pronoms, mais ils peuvent aussi rester implicites et dépendre de l'interprétation sémantique des énoncés. L'enchaînement perçu comme satisfaisant peut aussi être le produit d'un mixte de liens explicites et de liens implicites.

Les langues connaissent trois types distincts de liens : les liens anaphoriques, les liens méronymiques et les liens reformulatifs. Les deux premiers sont donnés dans la grammaire et la structure lexicale et sémantique de la langue, c'est-à-dire que le locuteur n'a pas la latitude de s'y soumettre ou non. S'il ne s'y soumet pas, son discours est incohérent. Par contre, le lien reformulatif est construit par le locuteur et, à condition de reprendre la forme d'un segment déjà produit, il jouit, en apparence, d'une marge assez grande de liberté.

Les liens anaphoriques apparaissent dans les matrices sous une référence unique : mentionné à tel endroit sous la forme $X$. Ce sont des liens homogènes, stables et, par définition, non ambigus. Les liens anaphoriques peuvent avoir un caractère méronymique et il n'y a pas, dans ce cas, de rupture franche entre anaphore et méronymie, mais les liens méronymiques couvrent globalement un champ spécifique au sein du discours et sont souvent indépendants de la grammaire de l'anaphore. La complexité liée à l'anaphore n'a d'outil de mesure dans les matrices analytiques définitoires que lorsqu'elle a partie liée avec la méronymie. Étant une forme de reconnaissance, elle ne présente, au regard de l'analyse matricielle, une forme ou une autre de complexité, que si sa reconnaissance exige de passer par des relations autres que les liens de reprise simple ou redondante qui en sont constitutifs. C'est le cas notamment lorsque l'antécédent n'est pas formé d'un individu mais d'une classe d'objets. Ainsi dans le passage suivant: Maillots de corps déchirés, pieds nus, le visage balafré, quelques 
vagues sacs à dos à l'épaule, la grande équipe regagnait en silence ses quartiers. L'expression grande équipe peut reprendre anaphoriquement " ceux qui ont des maillots déchirés, marchent pieds nus, ont le visage balafré, portent à l'épaule de vagues sacs à dos » du fait qu'ils sont des parties du tout qu'elle forme.

D'une matrice à l'autre, un lien est méronymique lorsqu'il porte sur les arguments internes ou sur la finalité de ces arguments ${ }^{38}$ tels qu'ils se manifestent dans la matrice d'un énoncé antérieur. Un énoncé est cohérent avec l'énoncé ou les énoncés qui le précèdent lorsque ses arguments internes partagent la finalité de ces énoncés antérieurs ou s'y réferent pour la contester ou en faire un prétexte ${ }^{39}$ à la nouvelle finalité qu'ils vont construire. Les relations entre "propositions " définies traditionnellement dans les grammaires françaises d'enseignement comme des relations de rection ou de subordination et même dans certains cas les relations de coordination $^{40}$ relèvent toutes finalement, à l'exception notable des relations de succession et de cooccurrence dans le temps (après, avant, depuis, ...), de liens qui ont, peu ou prou, une structure méronymique. Dire, en usant de connecteurs de subordination, Quand Zoé sera là, on en reparlera ou Je suis parti parce que je ne voulais pas le voir c'est dire, sans user de ces connecteurs, que La présence de Zoé dans notre conversation est un élément constitutif de la pertinence de cette conversation ou que Mon refus de le voir est un élément constitutif de mon

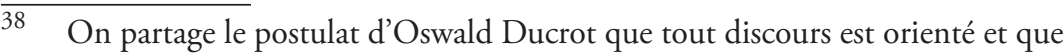
cette orientation est inscrite dans les propriétés lexicales des mots. La finalité d'une expression se confond souvent avec son deuxième argument interne. Ainsi si les arguments internes de porte sont fermeture PT passage on admettra que la finalité d'une porte est de permettre le passage. Par exemple, avec mais, pourtant ou puisque.

40 Souvent, la coordination, voire la juxtaposition de deux propriétés, présuppose nécessairement qu'elles ont vocation à être, par défaut ou par construction, des parties constitutives d'une même entité : Il est bête et méchant ou Il est bête mais adorable disent soit que le fait d'être bête entraîne chez le même individu - à défaut d'élément nouveau - le fait d'être méchant ou que, contrairement à ce qu'on pense - le personnage n'étant pas construit comme les autres, le fait d'être bête entraîne chez cet individu le fait d'être adorable. Dans les deux cas, la bêtise, la méchanceté ou le fait d'être adorables sont par définition - du point de vue de l'énonciateur qui fait de son point de vue un discours collectif évident de référence - les parties constitutives du tout dont il est question. Voir Amr Helmy Ibrahim, 1978. 
départ. Ou encore que Ce qu'on a à se dire n'a pas de sens si Zoé n'est pas là et qu'on ne peut comprendre mon départ qu'en sachant que je ne veux pas le voir.

Avec un connecteur comme quoique, le lien méronymique étend la perception de la cohérence à l'adhésion aux valeurs de l'énonciateur. Lorsque Voltaire écrit : (1) "Quoique vous soyez dans le printemps de votre vie (...) quoique vous soyez belle et que vos talents ajoutent à votre beauté, vous avez l'esprit très sage et le goût très fin et je vous ai entendue raisonner mieux que de vieux derviches à longue barbe et à bonnet pointu " ou "Quoique riche et jeune, il savait modérer ses passions; il n'affectait rien, il ne voulait point toujours avoir raison et savait respecter la faiblesse des hommes ${ }^{41}$ " il dit aussi : (1') femmes belles et talentueuses DC peu de chance d'être sage, d'avoir le goût fin et de savoir raisonner ou belle et talentueuse PT femme de goût et sachant raisonner; être riche et jeune DC ne pas modérer ses passions, être affecté, vouloir avoir toujours raison et ne pas respecter la faiblesse des hommes ou riche et jeune PT modérant ses passions, pas affecté, ne voulant pas toujours avoir raison, respectueux des faibles.

D'une matrice à l'autre, il y a reformulation selon deux modalités distinctes : lorsque la reformulation porte sur un ou plusieurs des actualisateurs du prédicat ou de la suite prédicative d'un énoncé ou des énoncés précédents ou lorsqu'elle porte sur le prédicat ou la suite prédicative elle-même.

Nous entendons par reformulation : "Tout processus de reprise d'un énoncé antérieur qui maintient, dans l'énoncé reformulé, une partie invariante à laquelle s'articule le reste de l'énoncé, partie variante par rapport à l'énoncé source ${ }^{42} »$. La reformulation étant un processus dynamique qui se trouve à cheval entre la reconstitution d'une équivalence à travers une

\footnotetext{
41 Voltaire, Zadig ou la destinée. Histoire orientale, Éditions de la Pléïade, Paris, Gallimard, 1747 [1979]. (consulté dans l'édition de la Pléiade, p. 55-57).

42 Claire Martinot, La reformulation dans les productions orales de définitions et explications (Enfants de maternelle), thèse de doctorat, Université Paris 8-SaintDenis, 1994 et «Quand Acquisition rime avec Reformulation : nécessité d'une réponse linguistique aux phénomènes d'acquisition de la langue maternelle ", Recherches linguistiques, ${ }^{\circ} 29,2007$, p. 179-212.
} 
formulation différente et la construction d'une formulation différente pour se démarquer de ce qui pourrait être perçu comme une équivalence, l'évaluation de ses effets est étroitement liée à la délimitation que l'on fait du segment invariant à l'intérieur de l'ensemble concerné par la reformulation.

Cet ensemble peut se réduire à une prédication simple reformulée en une autre prédication simple - par exemple, à l'intérieur d'un récit où il est question d'un personnage qui court vers une porte, quelqu'un dit Il a couru ouvrir la porte et est reformulé par un autre qui dit Il a couru fermer la porte où ouvrir est reformulé par fermer, l'invariant étant constitué par tout le reste de l'énoncé. Mais il peut aussi inclure des reformulations différentes d'un énoncé comportant une prédication principale et une ou plusieurs prédications secondes - par exemple, dans un contexte analogue au précédent : Boitant, tirant le petit derrière lui pour qu'il ne lui échappe pas, il a couru ouvrir la porte, reformulé en Il a couru, avec sa jambe qui boite et le petit qu'il serrait toujours pour qu'il ne s'enfuie pas, pour fermer la porte - où l'invariant est Il a couru (faire quelque chose concernant) la porte, tandis que différents types de reformulations portent sur l'ordre des constituants dans la construction de l'énoncé et les expressions boitant $>$ avec sa jambe qui boite; tirant le petit derrière lui pour qu'il ne lui échappe pas > et le petit qu'il serrait toujours pour qu'il ne s'enfuie pas; ouvrir > pour fermer.

Comme il peut porter sur des reformulations intriquées de plusieurs énoncés formant une unité de sens équivalente à un prédicat unique ou macro-prédicat - par exemple : Il n'a plus qu'une idée en tête, comment se sortir de là. Il court dans tous les sens. Les portes sont verrouillées. Il y a des barreaux aux fenêtres. Tout d'un coup une idée. La cheminée. Elle est assez large. Il grimpe. Il a peur. Il croit mourir. Mais il sent l'air frais sur son visage. Encore un effort, une écorchure, le chapeau de la cheminée à pousser. Et le voilà libre. Reformulé en Il faut sortir. Il court dans tous les sens. Il y a des cadenas sur toutes les portes, des barreaux aux fenêtres. Sauf une. Là haut. Il empile des chaises, grimpe, se blesse en cassant la vitre puis, passant son corps dans l'encadrement, il saute. Il a mal. 
Il s'est sûrement cassé quelque chose en tombant de si haut. Mais il est libre. - où l'invariant est l'unité de sens : Il veut sortir par n'importe quel moyen de l'espace où il se trouve. Il cherche et trouve un moyen. Il fournit un gros effort, souffre, se fait mal mais parvient à sortir.

On voit que, dans ce dernier cas, la délimitation formelle précise, donc linguistique, du segment invariant, n'est pas aisée et que, par conséquent, la définition de l'unité de sens portée par cet invariant peut à son tour prêter à controverse. La complexité concaténative liée à la reformulation peut alors devenir exponentielle du fait de l'ouverture de l'éventail des choix possibles et de la diversité des paramètres attachés à ces choix. Il n'en reste pas moins, et c'est probablement là la raison principale de la prédilection des enfants pour la reformulation comme outil d'appropriation de leur langue maternelle, comme le montrent les travaux de Claire Martinot et des membres de son équipe internationale ${ }^{43}$, qu'en reprenant un invariant, qu'il soit enregistré dans la mémoire sous la forme morphosyntaxique de son occurrence, sous sa représentation sémantique ou sous un mixte des deux, qu'il ait été " compris " ou non, la reformulation mesure en quelque sorte la complexité. En effet, une reformulation exhibe toujours les outils - mots ou constructions - qu' elle se donne pour définir et expliquer ce qu'elle reformule. Et elle le fait parce que, en réalité, celui qui reformule s'explique à luimême ce qu'il reformule pour se l'approprier. Or, ce procédé, théoriquement complexe du fait de la multiplicité des choix et des paramètres qu'il présuppose, est souvent chez l'enfant - plus rarement chez l'adulte - une procédure de décomplexification de l'invariant. Le procédé reste complexe chez l'adulte qui pratique une décomplexification le plus souvent informée et sélective, parfois même biaisée par la volonté de déformer sciemment les propos qu'il reformule. Souvent, chez l'adulte, le processus de décomplexification consciente par reformulation reprend à

$43 \quad$ Voir Claire Martinot, « Reformulations paraphrastiques et stades d'acquisition en français langue maternelle ", Cahiers de praxématique, n 52, 2009, p. 29-57. 
l'échelle d'une série de propositions, d'énoncés, voire d'un texte, une analyse qui rappelle par bien des égards, même si elle ne s'y réduit pas, la dégrammaticalisation que fait subir à un énoncé simple l'analyse matricielle définitoire.

Les deux premiers types de liens - anaphorique et méronymique - bien que plus ou moins fortement grammaticalisés ne s'affichent pas comme tels. Le locuteur fait le lien, mais ne signale pas qu'il le fait. Par contre, quand il reformule, le locuteur souligne souvent ce qu'il fait et détaille la dimension autonymique ou plus généralement métalinguistique de son énonciation. Prenons un exemple élémentaire :

(15) (On lui a fait un pontage + Il a été ponté). Il reprendra ses activités dans 3 ou 4 jours.

(16) (On a fait le pontage + Le pont est fait). Dans un mois le navire sera à flot.

(17) (On a fait un pontage + Un pont a été [jeté + établi]). Ils passeront à la faveur de la nuit.

On a fait $(u n+$ le) pontage revient en tête de (15), (16) et (17) mais sa matrice donne à chaque fois une description différente de pontage.

(15-m) Quelqu'un qui n'est pas désigné dit que des gens qui ne sont pas désignés ont (réalisé + installé + greffé) un conduit de passage du sang sous forme de pont entre deux veines obstruées.

(16-m) Quelqu'un qui n'est pas désigné dit que des gens qui ne sont pas désignés ont recouvert la partie découverte du bateau consacrée à la circulation entre ses points extrêmes qui s'appelle pont.

(17-m) Quelqu'un qui n'est pas désigné dit que des gens qui ne sont pas désignés ont (construit + établi + fait + jeté + réalisé) un pont pour le passage par dessus un obstacle séparant deux points. 
Dans (15-m), le prédicat pontage correspond aux arguments internes obstruées PT conduit de passage qui ont pour finalité reprise du passage du sang [obstruction et passage sont des corrélats].

Dans (16-m), le prédicat pontage correspond aux arguments internes couverture DC circulation qui ont pour finalité circulation entre les points extrêmes d'un bateau [couverture et circulation sont des corrélats].

Dans (17-m), le prédicat pontage correspond aux arguments internes obstacle PT passage par dessus qui ont pour finalité de relier deux points séparés jusque là par un obstacle [obstacle et passage sont des corrélats].

Si dans chacune des suites de deux énoncés 15,16 et 17 on substitue au deuxième énoncé de l'une, le deuxième énoncé d'une autre, on a un discours incohérent qui révèle immédiatement une incompréhension ou un usage fautif du prédicat.

$\mathrm{Si}$, maintenant on s'interroge sur les reformulations possibles sans changement de prédicat, on constatera qu'elles vont porter sur la redéfinition explicite des actualisateurs présents dans la matrice, qu'il s'agisse des supports, des verbes distributionnels sélectionnant une classe d'objets (greffer), des corrélats ou des noms génériques (point).

Les trois types de liens que nous avons évoqués - anaphorique, méronymique et reformulatif - ne sont pas nécessairement liés à des types de phrases. C'est probablement pourquoi la classification traditionnelle des grammaires françaises d'enseignement qui a longtemps distingué et distingue parfois toujours des phrases indépendantes, coordonnées, principales, subordonnées peut être trompeuse pour évaluer la simplicité ou la complexité d'un énoncé. Elle rend compte de liens morphologiquement marqués et qui ont, de ce fait, une pertinence formelle indiscutable mais ne rend souvent pas bien compte des relations de rection et de dépendance et encore moins de la nature de ces relations au regard de l'ensemble des relations grammaticales qui construisent le sens et définissent les limites effectives d'un énoncé simple. 


\subsection{Cas des relatives}

Toutes les langues qui connaissent les constructions qu'on appelle dans les langues indo-européennes relatives ${ }^{44}$, y distinguent, grosso modo, deux grands types correspondant à la bipartition que fait la tradition grammaticale française depuis Port-Royal : les déterminatives et les explicatives. Elles illustrent bien deux formes différentes de complexité : la complexité intégrative et la complexité concaténative externe. Les relatives déterminatives L'homme que tu m'as présenté hier est un traître ou en arabe moderne Al rajol alladhi qaddamtaho lî 'ams khầén - où la relative : que tu m'as présenté hier / 'alladhi qaddamtaho lî 'ams, n'est pas effaçable - relèvent de la complexité intégrative dans la mesure où elles participent de la complétude de l'énoncé simple et constituent une partie intégrante de sa matrice, alors que les relatives explicatives - Un homme que tu m'as présenté hier m'a trahi / Il y a un homme que tu m'as présenté hier qui m'a trahi ou en arabe moderne khânani rajol qaddamtaho lî 'ams / Honâka rajolon qaddamtaho lî 'ams khânani - où la relative : que tu m'as présenté hier / qaddamtaho lî 'ams, est effaçable sans que cela affecte la complétude ou la grammaticalité de l'énoncé - relèvent de la complexité concaténative externe dans la mesure où elles ont une matrice indépendante de la matrice de l'énoncé dans lequel elles s'enchâssent et que la grammaticalité et la bonne formation de cet enchâssement ne sont contraintes que par la compatibilité des arguments internes de l'énoncé d'accueil et de l'énoncé enchâssé ainsi que de leur finalité.

L'observation du fonctionnement des relatives dans cinq langues ou variétés de langue (français, polonais, anglais, arabe moderne et arabe égyptien) illustrera cette complexité. On y trouve 18 cas de figure possibles ( 3 fonctions principales pour le relatif [sujet, complément direct, complément indirect] x 3 cas de nombre [singulier, duel, pluriel] x 2 cas de genre [féminin, masculin] $=18$ ) et on constate que le choix des locuteurs s'exerce dans quatre champs.

44 La dénomination peut varier selon les langues. Par exemple, la tradition grammaticale arabe parle de maws:oul (= rattaché ou mis en connexion avec). 


\section{(1) La forme du pronom relatif :}

/1a/ Le choix de la forme du pronom relatif.

/1b/ S'il y a plus d'une forme disponible, le nombre de formes parmi lesquelles il faut choisir et le rapport des paramètres du choix à la forme choisie. Ainsi, une relation bijective qui impliquerait qu'à chaque combinaison de paramètres (fonction, nombre, genre) correspondrait une forme distincte, multiplierait les formes et la charge mémorielle qu'entraînerait cette multiplication, mais réduirait à zéro l'effort impliqué par le choix. La question est ouverte de savoir si, par rapport aux processus cognitifs cérébraux, l'effort de reconnaissance de la bonne forme parmi un grand nombre de formes monosémiques mémorisées est plus important ou moins important que l'effort de sélection de la bonne relation parmi un petit nombre de formes polysémiques dont les potentialités combinatoires sont plus ou moins grandes. La question est par contre réglée du point de vue du linguiste qui sait que les relations strictement bijectives constituent plutôt l'exception que la règle. Si on considère, comme nous l'avons suggéré, que l'effort mémoriel brut ne relève pas de la complexité, c'est-à-dire que le simple fait de retrouver dans un stock d'expressions, l'expression qui correspond à la relation bijective recherchée, quelle que soit la taille du stock, n'est pas une opération complexe, et que la complexité est proportionnelle à la charge du nombre de paramètres à intégrer dans un choix et au nombre de choix à faire, la situation sera d'autant plus complexe que le nombre de formes disponibles sera, à condition d'être supérieur à un, faible. En effet, quand le nombre de formes disponibles est faible, il y a nécessairement un nombre plus grand de configurations différentes qui utilisent les mêmes formes, donc un nombre plus important de choix à faire parmi les constructions possibles. Mais l'appréciation de ce rapport dans la perspective d'une comparaison des langues n'est pas automatique du fait que le nombre de combinaisons possibles n'est pas le même dans toutes les langues. Ainsi, en arabe les combinaisons possibles impliquant le nombre sont plus importantes du fait de l'existence d'un duel; en allemand, les combinaisons impliquant le genre 
sont plus importantes du fait de l'existence du neutre, en français, mais pas en anglais ou en polonais, les formes associant une préposition au pronom relatif sont plus nombreuses du fait qu'elles sont systématiquement dédoublées par référence au paramètre du genre (auquel ou à laquelle, duquel ou de laquelle, etc.); en polonais et en arabe classique ou moderne, l'obligation de marquer la fonction (terminaison casuelle) augmente certes les combinaisons possibles mais du fait qu'elle est en phase avec une contrainte grammaticale générale pour tous les constituants (langues casuelles) ne présente pas, comme c'est le cas en français (formes différentes suivant la fonction : qui, que, dont, où), et dans une moindre mesure en anglais (notamment who ou whom), un marquage exceptionnel. Si l'on considère qu'un choix qui ne suit pas une pratique générale est plus complexe qu'un choix qui la suit, alors le choix du pronom relatif approprié en français présente sur ce point un degré de complexité plus grand que le choix du pronom en polonais bien que l'éventail des formes possibles soit plus grand en polonais. Ce sont ces différences de parcours qui font que, d'une langue à l'autre, les différents types de complexités se compensent et qu'on aboutit en définitive à une sorte de péréquation des complexités.

Tous les choix que nous avons mentionnés font partie des opérations de quantification et de détermination qui interviennent lors de l'adéquation catégorielle. Ils accompagnent la mise en place de la grammaire. La complexité que ces choix doivent gérer est de type intégratif et se mesure au sein de la matrice analytique au nombre de mentions ayant soit la forme : $x$ désigne une manifestation de forme $z$ de y qui \{est connue + est prototypique de y + est un y générique + est un y quelconque $\}+\{a$ (une taille + un nombre + une quantité) (non spécifiée + correspondant à [un nombre d'unités de y + une partie p de y + un type t de $y]$ )\}, soit la forme La forme $z$ de $x$ désigne (celuilcelle/ceux qui fait $y+$ l'objet de $y+$ le champ d'application de $y+$ le lieu de $y+$ le responsable de y + le bénéficiaire de y + le témoin de y + la victime de $y+$ la source de $y+$ la destination de $y+$ une relation constante entre $x$ et $y$ ). 
La quantification et la détermination sont, dans toutes les langues du monde, incontournables. Il n'existe pas, à notre connaissance, de langue susceptible de produire des énoncés qui échapperaient à leur grammaire. L'universalité des relatives ou de ce qui en tient lieu vient du fait qu' elles parachèvent ou expliquent les opérations de détermination et de quantification grammaticalisées par la langue. Elles peuvent donc, en termes de compréhension, les rendre plus transparentes, c'est-à-dire qu'en révélant et en explicitant la succession des choix qui ont mené à leur grammaticalisation, elles mesurent la complexité de ces choix tout en défaisant leur complexité. Les analyser correctement au sein des matrices est donc essentiel à l'établissement d'un étalon indécomposable de la simplicité.

(2) L'existence ou non d'une proposition relative sans la présence d'un pronom relatif :

/2a/ La possibilité d'effacer, ou de réduire, le pronom relatif. Présente en anglais et dans les deux variétés d'arabe que nous avons observées, cette possibilité témoigne, quelles que soient les conséquences de sa réalisation, d'un certain niveau de complexité dans la mesure où le choix ne peut pas être esquivé, même si, comme en anglais, il peut s'agir d'une variante libre.

/2b/L'obligation d'effacer, ou de réduire, le pronom relatif : présente dans les deux variétés d'arabe, elle constitue un niveau élevé de complexité dans la mesure où ce choix contraint est indissolublement lié au système de la détermination.

Dans les deux cas il s'agit d'un processus de concaténation externe. La réduction ou le maintien d'une relative - donc l'aptitude à trouver la forme adéquate d'une détermination et à distinguer une déterminative d'une explicative - présuppose que tous les mécanismes nécessaires à la complétude de l'énoncé simple ont été parfaitement maîtrisés.

\section{(3) La reprise ou non de l'antécédent à l'intérieur de la pro- position relative (en plus du pronom relatif) :}

Ce phénomène est propre à l'arabe et s'inscrit dans une opposition typologique plus vaste des systèmes de pronominalisation 
très redondants des langues sémitiques - ou afro-asiatiques - par rapport aux langues indo-européennes et d'autres groupes de langues. On a là clairement un niveau de complexité pour le locuteur extérieur à la langue, mais on ne peut pas, tout aussi clairement, postuler un niveau de complexité pour le locuteur natif dans la mesure où il n'a pas de choix supplémentaire à faire du fait qu'il construit une relative. Plus généralement, on peut considérer que l'accroissement des redondances est dans un rapport inverse à l'accroissement de la complexité.

(4) La présence et la position d'une préposition liée à la fonction casuelle indirecte de l'antécédent :

/4a/ L'association d'une préposition au relatif lorsque le relatif a une fonction casuelle indirecte; là aussi cette possibilité oppose les deux variétés de l'arabe aux trois langues indo-européennes observées.

/4b/ La possibilité ou l'obligation de faire apparaître à l'intérieur de la proposition relative la préposition liée à la fonction casuelle indirecte du relatif; ici c'est clairement l'anglais qui, par les choix qu'il autorise, introduit un niveau indiscutable de complexité.

Comme dans le premier champ, on a affaire à un processus d'adéquation catégorielle et à une complexité de type intégratif mais avec une configuration différente dans la matrice. Les prépositions sont liées à la structure d'argument du prédicat et la description du type de grammaticalisation qu'elles cristallisent n'a pas, comme pour les opérations de quantification et de détermination, une forme fixe et universelle. La complexité dans ce cas est minimale pour les natifs - la distribution de la préposition est indissociable d'un usage grammatical du prédicat - mais peut être maximale pour les allophones. Il y a, en effet, peu de chances pour que la distribution des prépositions soit identique d'une langue à l'autre. L'analyse matricielle se trouve ici confrontée, en termes de comparaison des langues ou de typologie, à l'une de ses limites majeures. D'une part, elle ne signale pas de complexité particulière puisqu'elle se contente de rétablir les 
éventuelles ellipses de la structure prédicative; d'autre part, ce qui est peut-être plus problématique, elle n'a pas d'outil pour expliquer et encore moins justifier la position de la préposition dans l'ordre de succession des constituants de l'énoncé. Cet ordre ne présente pas d'importance ou de difficulté particulière lorsqu'il est libre ou qu'il est lié à un effet stylistique descriptible, mais il constitue une boîte noire au regard de l'analyse matricielle lorsqu'il est contraint. C'est pourquoi l'analyse matricielle n'a rien à dire sur, par exemple, la différence de placement des prépositions en anglais et en français. Le tableau 2 donne l'ensemble de nos observations.

Tableau 2

\begin{tabular}{|c|c|c|c|c|c|}
\hline & Fr & Po & An & AM & AE \\
\hline $\begin{array}{c}\text { I-Variation de forme du } \\
\text { pronom }\end{array}$ & + & + & + & + & - \\
\hline II- Nombre de variations & $4 / 18^{+}$ & $10 / 15^{+}$ & $4 / 9^{+}$ & 6 & 0 \\
\hline $\begin{array}{c}\text { III- Réduction choisie - Variante } \\
\text { libre }\end{array}$ & - & - & + & - & - \\
\hline $\begin{array}{c}\text { IV- Réduction obligatoire et } \\
\text { conditionnée du pronom }\end{array}$ & - & - & - & + & + \\
\hline $\begin{array}{c}\text { V- Reprise de l'antécédent par } \\
\text { un pronom dans la relative }\end{array}$ & - & - & - & + & + \\
\hline $\begin{array}{c}\text { VI- Association d'une } \\
\text { préposition au pronom relatif }\end{array}$ & + & + & + & - & - \\
\hline $\begin{array}{c}\text { VII -Apparition possible d'une } \\
\text { préposition dans la relative }\end{array}$ & - & - & + & + & + \\
\hline
\end{tabular}

$\mathrm{Fr}=$ français, $\mathrm{Po}=$ polonais, $\mathrm{An}=$ anglais, $\mathrm{AM}=$ arabe moderne, $\mathrm{AE}=$ arabe égyptien

La barre oblique sépare les formes simples du pronom relatif (à gauche) des formes associées à des prépositions élémentaires. Le + en exposant indique que l'association peut se faire avec d'autres prépositions ou locutions prépositives. Il faut entendre par " relative ": " proposition relative ", par " pronom ": " pronom relatif ".

En termes de choix, et compte tenu de ce que nous avons avancé plus haut, on voit que le système du relatif est, dans sa spécificité, le moins complexe en arabe égyptien (3 choix : IV, V $\&$ VII) et le plus complexe en anglais (6 choix : I, II (2), III, VI 
\& VII). Le français avec 4 choix (I, II (2), VI) est moins complexe que l'anglais ou l'arabe moderne ( 5 choix : I, II, IV, V, VII), mais aussi complexe que le polonais (4 choix : I, II (2), VI).

En termes de nature du choix, c'est-à-dire de sa difficulté intrinsèque résultant du rapport entre le nombre de paramètres à intégrer et le nombre de formes disponibles, la réponse dépend directement de la théorie de la grammaire (au sens d'un ensemble réglé de relations) que l'on se donne. Celle-ci se doit, sauf à penser que les locuteurs des différentes langues appartiennent à des espèces humaines différentes, d'être universelle et de s'appuyer pour l'analyse de chaque phénomène observé, quelle que soit sa complexité, sur des mécanismes au départ au moins aussi simples que ceux que l'on peut rencontrer pour exprimer ce phénomène dans n'importe laquelle des sept mille langues du monde.

\section{Conclusion}

Les mécanismes que nous venons de décrire sont stables, polyvalents et exprimables dans n'importe quelle langue. Ils ne sont pas propres à un moment particulier de l'évolution. Ce sont des outils de description et d'explication, mais ce sont aussi des facteurs d'évolution. Les choix de reconstitution et de réduction que fait le locuteur ont une incidence directe sur le sens des formes qu'il utilise.

Les langues ne peuvent pas construire, moduler et faire évoluer le sens uniquement ni même essentiellement par des associations bijectives entre une forme et un contenu du fait que nous sommes soumis à des impératifs de capacité mémorielle, d'économie et de changement. Elles ne peuvent pas non plus confier la construction du sens à une combinatoire ouverte de composants élémentaires dont l'explosion combinatoire deviendrait vite ingérable. Elles doivent donc construire des grammaires, c'est-àdire des systèmes de relations différenciées, autorégulées et finies sans être figées. Les parcours d'élaboration de ces grammaires comportent des constantes, mais sont de fait très différents, notamment dans la manière avec laquelle ils se soumettent aux impératifs d'économie et accèdent à une forme ou à une autre 
d'efficacité expressive et dans la manière aussi avec laquelle ils préservent les possibilités créatives et combinatoires des constituants qu'ils ont utilisés. Enfin, ces parcours, lorsque la construction de la grammaire est suffisamment achevée pour être opératoire, ne sont visibles que par une déconstruction de ces grammaires. Cette déconstruction, pour être " traçable " - donc reproductible - doit utiliser le matériau qui a construit la grammaire. Les mots et les relations utilisés pour construire le sens doivent se retrouver dans sa déconstruction et sa reconstruction. Les relations grammaticales peuvent avoir été choisies ou construites arbitrairement - d'où les différences grammaticales entre les langues -, mais elles sont toujours un abrègement particulier d'un discours sensé exprimable dans n'importe quelle langue. Ce discours est la matrice analytique définitoire de toutes les langues et la complexité d'un énoncé observable dans une langue particulière est isomorphe à la complexité des paramètres parmi lesquels un locuteur choisit les étapes et les opérations nécessaires pour passer de cette matrice à l'énoncé observé.

Lactivité du locuteur est ainsi homogène et mesurable, car il reconstitue ou réduit les descripteurs des relations grammaticales à l'œuvre dans l'énoncé en même temps qu'il se donne une formulation simplifiée et étendue de ce que signifie l'énoncé qưil produit ou qu'il comprend. L'outil de mesure change de forme et de niveau, mais il ne cesse jamais de faire partie de l'objet mesuré. La complexité ressort donc de la manière avec laquelle la langue décrit elle-même l'usage qui en est fait. 


\section{Bibliographie}

Berthoz, Alain, La simplexité, Paris, Odile Jacob.

Carel, Marion, "Argumentation interne et argumentation externe au lexique : des propriétés différentes ", dans Amr Helmy Ibrahim (dir.), Langages, Les discours internes au lexique, $\mathrm{n}^{\circ}$ 142, Paris, Larousse, 2001, p. 10-21.

Ducrot, Oswald, 2001, "Critères argumentatifs et analyse lexicale ", Dans Amr Helmy Ibrahim (dir.), Langages, Les discours intérieurs au lexique, $n^{\circ}$ 142, Paris : Larousse, 2001, p. 22-40.

Gheerbrant, Frédérique (Yannick), La nominalisation et les verbes de sentiment, Thèse de doctorat de $3^{\mathrm{e}}$ cycle, Université Paris 7, 1978, 323 p.

Gould, Stephen Jay et Elizabeth S. Vrba, «Exaptation - A missing term in the science of form ", Paleobiology, vol. 8, no 1, 1982, p. 4-15.

Gross, Gaston, Les constructions converses du français, Genève-Paris, Droz, 1989.

Gross, Maurice, "Les verbes supports d'adjectifs et le passif ", dans Amr Helmy Ibrahim (dir.), Langages, Les supports, $\mathrm{n}^{\circ} 121$, Paris, Larousse. 1996, p. 8-18.

Gross, Maurice, Méthodes en syntaxe : régime des constructions complétives, Paris, Hermann, 1975.

Gross, Maurice, "Une grammaire locale de l'expression des sentiments ", Langue française, vol. 105, 1995, p. 70-87.

Hockett, Charles F., «The Origin of Speech », Scientific American, 203/3, 1960, p. 89-97.

Humboldt, Wilhelm von, " La recherche linguistique comparative dans son rapport aux différentes phases du développement du langage ", Introduction à l'ouvre sur le kavi et autres essais, [Traduction française par Pierre Caussat d'un mémoire lu par Humboldt à l'Académie de Berlin le 29 juin 1820], Paris, Seuil, [1820], 1974, p. 71-96.

Ibrahim, Amr Helmy, Coordonner pour argumenter, Semantikos vol. 2, $n^{\circ} 3 / 4$, Paris : The Semantikos Association, 1978, p. 21-42.

Ibrahim, Amr Helmy, Étude comparée des systèmes verbaux de l'arabe égyptien, de l'arabe moderne et du français, Thèse de doctorat d'Etat, Université Paris 7, 1979.

Ibrahim, Amr Helmy, « Peut-on reconnaître automatiquement les supports du non-fini en français et en arabe? », BULAG (Bulletin de Linguistique Appliquée et Générale), $\mathrm{n}^{\circ}$ 23, Besançon, Université de FrancheComté, 1998, p. 245-273. 
Ibrahim, Amr Helmy, "Une classification des verbes en 6 classes asymétriques hiérarchisées ", Syntaxe et Sémantique 2, dans Françoise Cordier, Jacques François et Bernard Victorri (dir.), Sémantique du lexique verbal, Caen, Presses universitaires de Caen, 2000, p. 81-98.

Ibrahim, Amr Helmy, "Argumentation interne et enchaînements dans les matrices définitoires ", dans Amr Helmy Ibrahim (dir.), Langages. Les discours intérieurs au lexique, $\mathrm{n}^{\circ}$ 142, 2001, p. 92-126.

Ibrahim, Amr Helmy, "Prolégomènes à une typologie de l'actualisation des noms ", dans Jacques François et Imtraud Behr (dir.), Les constituants prédicatifs et la diversité des langues, Mémoires de la Société de linguistique de Paris, Tome XIV, 2004, p. 29-76.

Ibrahim, Amr Helmy, « Les perturbations des relations d'appropriation et de détermination liées à l'actualisation des noms sont-elles réajustables? ", dans Béatrice Vaxelaire, Rudolph Sock, Georges Kleiber et Fabrice Marsac (dir.), Perturbations et réajustements : langue et langage, Strasbourg, Publications de l'Université Marc Bloch- Strasbourg 2, 2007, p. 279-286.

Ibrahim, Amr Helmy, "Moment, phase et manière du procès dans les matrices analytiques définitoires ", Philologica, Zbornik Filozofickej Fakulty Univerzity Komenského (Recueil de travaux de la Faculté des Lettres de l'Université Comenius), Bratislava, 2008, p. 83-93.

Ibrahim, Amr Helmy, "Les conditions de la prédication dans les langues ", Prédicats, prédication et structures prédicatives, Paris, CRL, 2009, p. 12-49.

Ibrahim, Amr Helmy, "Supports d'actualisation et dualité constitutive de la prédication ", Supports et prédicats non verbaux dans les langues $d u$ monde, Paris, CRL, 2010, p. 36-73.

Ibrahim, Amr Helmy, L'analyse matricielle définitoire : un modèle pour la description et la comparaison des langues, à paraître.

Ibrahim, Amr Helmy, "Le futur : une notion temporelle que les langues construisent avec de l'aspect ", dans Louis Begioni (dir.), Cluj-Napoca (Roumanie), Presses Universitaires de Cluj, à paraître.

Ibrahim, Amr Helmy, "Le futur : une notion temporelle que les langues construisent avec de l'aspect ", à paraître.

Martinet, André, Éléments de linguistique générale, Paris, Armand Colin, 2003 [1960].

Martinot, Claire, La reformulation dans les productions orales de définitions et explications (Enfants de maternelle), Thèse de doctorat, Université Paris 8-Saint-Denis, 1994. 
Martinot, Claire, «Quand Acquisition rime avec Reformulation : nécessité d'une réponse linguistique aux phénomènes d'acquisition de la langue maternelle ", Recherches linguistiques, n 29, 2007, p. 179-212.

Martinot, Claire, "Reformulations paraphrastiques et stades d'acquisition en français langue maternelle ", Cahiers de praxématique, n 52, 2009, p. 29-57.

Sarraute, Nathalie, Pour un oui ou pour un non, Paris, Édition de Poche, Folio Théâtre, 1999, [1982].

Voltaire, Zadig ou la destinée. Histoire orientale, Éditions de la Pléïade, Paris, Gallimard, 1747 [1979]. 